\begin{abstract}
Title of Thesis: $\quad$ SMALL FLUCTUATIONS IN EPITAXIAL GROWTH VIA CONSERVATIVE NOISE

Paul Patrone, Masters Degree, 2012

Thesis directed by: Professor Dionisios Margetis Department of Math
\end{abstract}

We study the combined effect of growth (material deposition from above) and nearest-neighbor entropic and force-dipole interactions in a stochastically perturbed system of $N$ line defects (steps) on a vicinal crystal surface in $1+1$ dimensions. First, we formulate a general model of conservative white noise, and we derive simplified formulas for the terrace width distribution (TWD) and pair correlations, particularly the covariance matrix of terrace widths, in the limit $N \rightarrow \infty$ for small step fluctuations. Second, we apply our formalism to two specific noise models which stem, respectively, from: (i) the fluctuation-dissipation theorem for diffusion of adsorbed atoms; and (ii) the phenomenological consideration of deposition-fluxinduced asymmetric attachment and detachment of atoms at step edges. We discuss implications of our analysis, particularly the narrowing of the TWD with the deposition flux, connection of noise structure to terrace width correlations, behavior of these correlations in the macroscopic limit, and comparison of our perturbation results to a known mean field approach. 


\title{
SMALL FLUCTUATIONS IN EPITAXIAL GROWTH VIA CONSERVATIVE NOISE
}

\author{
by \\ Paul Patrone \\ Thesis submitted to the Faculty of the Graduate School of the \\ University of Maryland, College Park in partial fulfillment \\ of the requirements for the \\ Masters Degree \\ 2012
}

Advisory Committee:

Professor Dionisios Margetis, Chair/Advisor

Professor Theodore Einstein, Co-Advisor

Dr. Gregg Gallatin 


\section{Acknowledgments}

I acknowledges support under the National Institute of Standards and Tech-

nology American Recovery and Reinvestment Act Measurement Science and Engineering Fellowship Program Award 70NANB10H026 through the University of Maryland; and by the NSF under MRSEC grant DMR 0520471 at the University of Maryland. 


\section{Table of Contents}

1 Introduction 1

2 Formulation and general results in $1+1$ dimensions $\quad 7$

2.1 Deterministic model . . . . . . . . . . . . . . . . . . 7

2.2 Stochastically perturbed system and linearization . . . . . . . . . . 10

2.3 TWD and pair correlations: general formulas . . . . . . . . . . . . . . 12

2.4 Covariance matrix: large- $N$ limit . . . . . . . . . . . . . . . 13

3 Particular cases of noise 16

3.1 Adatom noise under fluctuation-dissipation theorem . . . . . . . . . 16

3.2 Deposition-flux-induced noise at steps . . . . . . . . . . . . . . 20

4 Discussion 28

4.1 Noise structure and correlations . . . . . . . . . . . . . . . . 28

4.2 Mean field approach and decorrelation hypothesis . . . . . . . . . . 30

4.3 Related past works and plausible connection to experiments . . . . . 32

4.4 On the validity of the model . . . . . . . . . . . . . . . . . 34

4.5 Conclusion . . . . . . . . . . . . . . . . 36

$\begin{array}{ll}\text { A Derivations for covariant matrix } & 38\end{array}$

A.1 Useful relations . . . . . . . . . . . . . . . . . . . . . . . . 40

A.2 Long-time behavior of integral (2.13) . . . . . . . . . . . . 40

A.3 Two formulas . . . . . . . . . . . . . . . . . . . . . . . 41

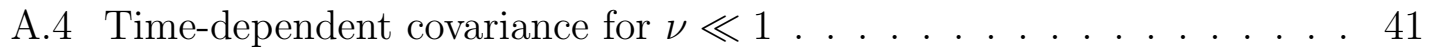

Bibliography 44 


\section{Chapter 1}

\section{Introduction}

A central question in nonequilibrium statistical mechanics is: how do largescale evolution laws emerge from the deterministic or stochastic dynamics of manyparticle systems [1]? Descriptions of linkages of particle models to full continuum theories lie at the heart of computational physics [2]. Related themes of modeling and analysis date back to classic works for the Boltzmann equation; see, e.g., [3-6].

Epitaxial growth consists of out-of-equilibrium processes that occur on crystal surfaces, usually in the presence of material deposition from above. Such processes accompany the fabrication of nanoscale structures, e.g., quantum wires and dots [7]. In particular, vicinal surfaces are characterized by nanoscale terraces oriented in the high-symmetry direction; the terraces are separated by line defects (steps) of atomic size. For macroscale surface regions at not-too-high temperatures, the steps can be considered as monotone (of the same 'sign'), with their number fixed by the miscut angle set by the experiment [8].

In this article, we address the question: how do terraces fluctuate on a macroscopic vicinal surface in $1+1$ dimensions? Our goal is to study the joint effect on the macroscopic behavior of noise and (microscopic) interactions of $N$ steps in the presence of growth. The noise is white and conservative, and is subject to geometric-type constraints: the total length of the sample does not fluctuate and the covariance of 
terrace widths is finite. Although our formalism is reasonably general for $1+1$ dimensions, we restrict attention to two specific models consistent with our constraints. One noise model comes from an application of the fluctuation-dissipation theorem to the density of adsorbed atoms (adatoms) [9]. Another model phenomenologically accounts for deposition-flux-induced fluctuations in the number of atoms that stick to steps [10]. For small fluctuations, the resulting stochastic differential equations (SDEs) are linearized around the average terrace width. We solve these equations, and describe the terrace width distribution or density (TWD) and terrace width pair correlations as $N \rightarrow \infty$.

By the Burton-Cabrera-Frank (BCF) theory [11], the major kinetic processes incorporated into the deterministic model are: (a) diffusion of adatoms on terraces; (b) attachment and detachment of atoms at step edges; and (c) external deposition with (given) flux, F. To simplify the analysis, we consider straight (kink free) steps and impose diffusion-limited (DL) kinetics, in which the diffusion of adatoms on terraces is the slowest process. In this case, the adatom density at each step edge attains an equilibrium value [8]. In addition, the steps are allowed to interact entropically and as force dipoles $[12,13]$.

Our analysis forms part of a relatively long sequence of works in stochastic step dynamics. The setting was motivated by the experimental issue how to control the TWD by material deposition $[14,15]$. Elements of our analysis were inspired by $[9,16]$. In particular, [16] predicted the narrowing of the TWD during growth via ad hoc white noise, neglect of step interactions, and application of a mean field approximation in $1+1$ dimensions. This prediction compared favorably with kinetic 
Monte Carlo simulations in two space dimensions (2D) [16].

Earlier systematic theories, e.g., $[9,17,18]$, make use of the fluctuation-dissipation theorem for the adatom density and flux in order to determine the noise, invoke richer step energetics and kinetics, and focus on effects of step meandering in $2+1$ dimensions. In particular, in [9] the authors point out that sufficient increase of the deposition flux causes a morphological step instability driven by adatom diffusion, which is distinct from effects of statistical fluctuations. Other studies include the phenomenological approaches of $[10,19]$ (see also [20]), where the noise models appear to have an empirical character.

Here, we aim to enrich the analysis of [16] in three ways. First, we include step interactions jointly with material deposition from above. Second, we use different models of noise. For instance, we account for a conservative noise consistent with the fluctuation-dissipation theorem in the spirit of [9]. As an alternative, we entertain the scenario of an F-dependent conservative white noise, following [10]. We show how these choices may tone down the $F$-dependence of the TWD predicted in [16]. Third, we describe terrace width pair correlations, thus not limiting our attention to the TWD. However, we leave out step meandering at this stage, in contrast to the more realistic (yet much more elaborate) treatment of $[9,17,18]$. One of our purposes is to quantify the connection of noise structure to terrace width correlations at both the microscale and the macroscale in a setting sufficiently simple, i.e., in one space dimension (1D), to enable tractable computations.

Despite its 1D character, our linearized stochastic model with interactions can be viewed as prototypical for a class of asymmetric discrete processes in the 
limit of large $N$. The kinetic process for the motion of terraces is asymmetric because of a drift (average lateral step velocity) proportional to $F$ (see section 2.2) $[16,21,22]$. This convective effect, although negligibly small in many material systems, is retained here for the sake of a more general treatment (in 1D). As a result, on every terrace the flux of deposited atoms to an upstep is different from the flux at a downstep. Similar kinetic effects arise from the Ehrlich-Schwoebel barrier [9, 23-27], electromigration currents [28-30], differences of atomistic origin in attachment rates $[26,31]$, and impurities $[32,33]$.

From a kinetic theory view, our work forms an extension of the treatment in [34-36] where the mean field approach of [16] is placed on the grounds of BogoliubovBorn-Green-Kirkwood-Yvon (BBGKY) hierachies for joint probability densities of terrace widths. In [34], the steps are energetically non-interacting, and the white noise is non-conservative. In [35], the steps are interacting, albeit in the absence of deposition (with $F=0$ ). Both step interactions and nonzero flux $F$ are discussed briefly in [36], which only reports (without derivations) the TWD from an ad hoc second-order conservative noise. Here, we consider different, physically-inspired and more transparent models of noise and, in addition, describe terrace width pair correlations. Moreover, we discuss discrepancies of some of our results from a mean field outcome.

Assuming small fluctuations of each terrace, induced by "small" noise terms, we linearize the SDEs. The ensuing terrace width stochastic process is Gaussian. The average terrace width is fixed at its initial value (consistent with the misorientation of the vicinal surface). We compute the terrace width covariance matrix 
analytically by allowing $N \rightarrow \infty$ while keeping the time $t$ independent of $N$ (see chapter 3). In particular, we derive relatively simple formulas for the terrace width covariance matrix in the steady state. By comparing our result for the terrace width variance to the mean field approach introduced in [16] and further discussed in [34], we indicate the role of the noise structure in terrace width pair correlations in the steady state; see sections 4.1 and 4.2. Plausible implications of our predictions are discussed in section 4.3 .

Our model is limited in its applicability. One limitation is due to the assumed 1D geometry. Because steps are straight, meandering is suppressed, and the noise for terrace widths has a relatively simple form. This setting contrasts the $2 \mathrm{D}$ geometry invoked e.g., in $[9,17,18]$. For instance, in $[9,18]$ Langevin forces are added to both the adatom diffusion equation and the boundary conditions for atom attachmentdetachment at curved steps. In [17], the noise is white in both time and the space coordinate along the step edge. Here, we resort to a tradeoff. On one hand, we analyze a tractable geometry. On the other hand, we exclude some realistic effects such as step meandering.

Another limitation, which is a consequence of linearizing step interactions for small fluctuations, is that steps can cross. This feature is, of course, unphysical. However, it has a negligibly small likelihood provided the TWD variance is small (see section 4.4).

Throughout this paper, we assume familiarity of the reader with basic concepts of epitaxial systems. For reviews on the subject, the reader may consult, e.g., $[7,8,37-39]$. 
The remainder of our paper is organized as follows. In chapter 2, we formulate generally the SDEs for 1D step motion in the presence of growth, step interactions and conservative noise. We solve the linearized SDEs and give general formulas for the TWD and terrace width covariance matrix. In chapter 3 , we compute the TWD and terrace width covariance matrix under two specific choices for the noise. In chapter 4, we discuss implications of our results: relation of noise structure to correlations, comparison to a mean field approach, validity of our model, and plausible connections to experiment. The appendices provide technical details needed in the main text.

Notation and terminology. We apply the Einstein summation convention, in which summation is implied by repeated indices, unless we state otherwise. The symbol $\mathcal{B}(t)$ denotes Brownian motion, while $\eta(t)=\mathrm{d} \mathcal{B} / \mathrm{d} t$ is white noise (where the time derivative is interpreted in the sense of distributions). The symbol $\mathbb{E}[X]$ denotes the average of the random variable $X$; the terms "mean" and "expectation" are also used for $\mathbb{E}$ interchangeably. Matrices and vectors are boldface. The matrix $\boldsymbol{C}$ is represented by $C_{k, l}$, indicating the entry at the $k$ th row and $l$ th column. The norm squared of the $N \times N$ circulant matrix $\boldsymbol{C}$ is $|\boldsymbol{C}|^{2}=\sum_{l=0}^{N-1}\left|C_{0, l}\right|^{2}=\sum_{k=0}^{N-1}\left|C_{k, 0}\right|^{2}$. By $f=\mathcal{O}(g)$ we imply that $f / g$ is bounded as a parameter or variable approaches an extreme value. The term "deposition flux" refers to $F$, i.e. material deposition from above; and "adatom flux" refers to the current due to diffusion of adatoms on terraces. 


\section{Chapter 2}

\section{Formulation and general results in $1+1$ dimensions}

In this section, we formulate SDEs for terrace widths in $1+1$ dimensions. Adatom diffusion is the main process driving the motion of steps by mass conservation. Atoms that migrate to the terrace boundaries attach to or detach from step edges; as a result, steps advance or retreat. Force-dipole and entropic step interactions (between nearest-neighbor steps) determine the equilibrium adatom density at each step edge, which in turn influences the net mass flux toward a step.

Because we consider continuous adatom densities, we also introduce Gaussian noise to account for microscopic deviations from continuum motion [40]. For a sufficiently weak noise term, we linearize the SDEs and derive general formulas for the TWD and terrace width covariance matrix.

\subsection{Deterministic model}

Let us begin with elements of the deterministic motion of terraces and steps. The geometry consists of straight steps of height $a$ at $x=x_{i}(t)$ (see figure 2.1). The $i$ th terrace is the region $x_{i}<x<x_{i+1}$, where $w_{i}(t):=x_{i+1}(t)-x_{i}(t)>0$ and $i=0, \ldots, N-1$. Apply screw periodic boundary conditions so that steps are mapped onto point particles on a ring. We set $w_{i}(0)=\varpi$, where $\varpi$ is a constant.

The formulation of equations for $x_{i}(t)$ is outlined in [34], and summarized here 


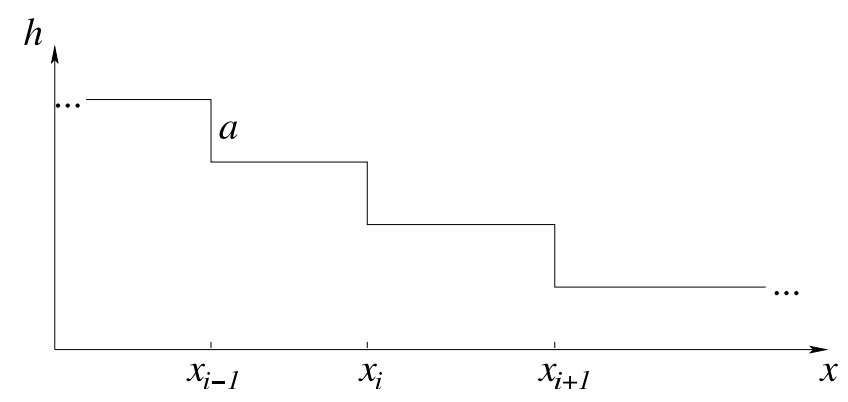

Figure 2.1: Schematic (cross section) of step geometry: $x=x_{i}(t)$ is the $i$ th step position, $a$ is the step height, and $h$ is the surface height.

with a more precise description of step interactions. In the presence of material deposition from above, steps have a typical (drift) velocity $v=F a \varpi$, where $F$ is the deposition rate. By a Galilean transformation to the comoving frame [16, 22], the adatom concentration $\varrho_{i}(x, t)$ on the $i$ th terrace satisfies $\left(D \partial_{\widetilde{x}}^{2}+v \partial_{\widetilde{x}}\right) \varrho_{i}+F=\partial_{\tilde{t}} \varrho_{i}$, where $D$ is the terrace diffusion constant and $(\widetilde{x}, \widetilde{t})=(x-v t, t)$. By the quasi-steady approximation we set $\partial_{\hat{t}} \varrho_{i} \approx 0$, which holds if deviations of the actual step velocity from $v$ are much smaller than the diffusive speed $D / \varpi$. Now remove the tildes for ease of notation $(\widetilde{x} \rightarrow x)$.

By linear kinetics, the atom attachment-detachment at the steps bounding the $i$ th terrace is expressed by $[8]-J_{i}\left(x_{i}\right)=\kappa\left[\varrho_{i}\left(x_{i}\right)-\varrho_{i}^{\mathrm{eq}}\right]$ and $J_{i}\left(x_{i+1}\right)=\kappa\left[\varrho_{i}\left(x_{i+1}\right)-\right.$ $\left.\varrho_{i+1}^{\text {eq }}\right]$, where $J_{i}(x)=-D \partial_{x} \varrho_{i}-v \varrho_{i}$ is adatom flux on the $i$ th terrace, $\varrho_{i}^{\text {eq }}$ is the equilibrium adatom concentration at the $i$ th step edge, and $\kappa$ is a constant rate. The quantity $\varrho_{i}^{\text {eq }}$ encapsulates energetics, e.g., force dipole step interactions $[8,12,13]$. Distinct rates $\kappa_{\mathrm{u}}, \kappa_{\mathrm{d}}$ for up- and down-step edges (Ehrlich-Schwoebel effect [23]) can also be included.

We enforce the conditions $v / \kappa \ll 1$ and $D / \kappa \ll \varpi$, which amount to DL 
kinetics. ${ }^{10}$ This means that we formally let $\kappa \rightarrow \infty$ in the attachment-detachment conditions at step edges, so that $\varrho_{i}\left(x_{i}\right) \rightarrow \varrho_{i}^{\text {eq }}$ since the flux is finite [16].

By mass conservation, each step advances or retreats in response to the net mass flux incident on it. Thus, the step velocity reads $\dot{x}_{i}=\mathrm{d} x_{i} / \mathrm{d} t=(\Omega / a)\left[J_{i-1}\left(x_{i}\right)-\right.$ $\left.J_{i}\left(x_{i}\right)\right]$ where $\Omega$ is the atomic area, $\Omega \approx a^{2}$. By solving the diffusion equation for $\varrho_{i}$ (treating the positions $x_{i}$ and densities $\varrho_{i}^{\text {eq }}$ as fixed), and thus determining $J_{i}(x)$, we obtain a system of ordinary differential equations (ODEs) for $x_{i}(t)$, and in turn for $w_{i}(t)[34]$ :

$$
\begin{aligned}
\dot{w}_{i}= & \frac{\mathrm{d} w_{i}}{\mathrm{~d} t}=\frac{a F}{2}\left\{\frac{w_{i+1} e^{\frac{v w_{i+1}}{2 D}}}{\sinh \left(\frac{v w_{i+1}}{2 D}\right)}-\frac{2 w_{i} \cosh \left(\frac{v w_{i}}{2 D}\right)}{\sinh \left(\frac{v w_{i}}{2 D}\right)}+\frac{w_{i-1} e^{\frac{-v w_{i-1}}{2 D}}}{\sinh \left(\frac{v w_{i-1}}{2 D}\right)}\right. \\
+ & a \varpi \\
& {\left[\varrho_{i+2}^{\mathrm{eq}} \frac{e^{\frac{v w_{i+1}}{2 D}}}{\sinh \left(\frac{v w_{i+1}}{2 D}\right)}-\varrho_{i+1}^{\mathrm{eq}}\left(\frac{e^{-\frac{v w_{i+1}}{2 D}}}{\sinh \left(\frac{v w_{i+1}}{2 D}\right)}+\frac{2 e^{\frac{v w_{i}}{2 D}}}{\sinh \left(\frac{v w_{i}}{2 D}\right)}\right)\right.} \\
& \left.\left.+\varrho_{i}^{\mathrm{eq}}\left(\frac{2 e^{-\frac{v w_{i}}{2 D}}}{\sinh \left(\frac{v w_{i}}{2 D}\right)}+\frac{e^{\frac{v w_{i-1}}{2 D}}}{\sinh \left(\frac{v w_{i-1}}{2 D}\right)}\right)-\varrho_{i-1}^{\mathrm{eq}} \frac{e^{-\frac{v w_{i-1}}{2 D}}}{\sinh \left(\frac{v w_{i-1}}{2 D}\right)}\right]\right\},
\end{aligned}
$$

where $i=0,1, \ldots, N-1$. It remains to express each $\varrho_{i}^{\text {eq }}$ in terms of positions $x_{i}$.

The step interactions are introduced explicitly in the $i$ th-step chemical potential, $\mu_{i}$, through the relation $\varrho_{i}^{\mathrm{eq}}=\rho_{0}\left(1+\mu_{i} / T\right)$ where $\rho_{0}$ is the equilibrium adatom density on a terrace, and $T$ is the Boltzmann energy [8]. If $E_{N}\left(\left\{x_{i}\right\}\right)$ is the total energy per unit length of the step train, we have $\mu_{i}=\Omega\left(\partial E_{N} / \partial x_{i}\right)$. For entropic and force-dipole interactions of strength $\breve{g}$, this $E_{N}$ reads $[8,12,13]$

$$
E_{N}=\breve{g} \sum_{i}\left(\frac{a}{w_{i}}\right)^{2} \Rightarrow \mu_{i}=\breve{g} a\left[\left(\frac{a}{w_{i}}\right)^{3}-\left(\frac{a}{w_{i-1}}\right)^{3}\right] \quad(\breve{g}>0) \text {. }
$$

Equation (2.1) is rewritten accordingly by eliminating all of the $\varrho_{i}^{\text {eq }}$ in favor of $w_{i}$. We leave this task to the interested reader. If $w_{i}(0)=\varpi$, then $w_{i}(t) \equiv \varpi$ is a stable

\footnotetext{
${ }^{01}$ For $v=\mathcal{O}(1)>0$ a more precise condition on $v$ reads $v / \kappa \ll \tanh [v \varpi /(2 D)]$.
} 
(equilibrium) solution for all $t>0$.

\subsection{Stochastically perturbed system and linearization}

Next, we add noise to ODEs (2.1) by writing

$$
\dot{w}_{i}(t)=\widetilde{A}_{i}\left(w_{i-2}, w_{i-1}, w_{i}, w_{i+1}, w_{i+2}\right)+\varpi \widetilde{Q}_{i, j} \widetilde{\eta}_{j}, \quad w_{i}(0)=\varpi
$$

where $\widetilde{A}_{i}\left(w_{i-2}, w_{i-1}, w_{i}, w_{i+1}, w_{i+2}\right)$ is the right hand side of $(2.1)$ in view of $(2.2)$; the matrix $\widetilde{\boldsymbol{Q}}$ is circulant and has units of time raised to the power $-1 / 2$; and $\widetilde{\boldsymbol{\eta}}$ is a vector Gaussian white noise, i.e., $\widetilde{\eta}_{i}=\mathrm{d} \mathcal{B}_{i} / \mathrm{d} t$. The matrix $\widetilde{\boldsymbol{Q}}$ in principle depends on $\left\{w_{i}\right\}$ and $\left\{\widetilde{\eta}_{i}\right\}$, but here we consider only constant $\widetilde{\boldsymbol{Q}}$. The factor $\varpi$ multiplying $\widetilde{\boldsymbol{Q}}$ has been extracted for later algebraic convenience. The components $\widetilde{\eta}_{j}$ obey the relation $\mathbb{E}\left[\widetilde{\eta}_{i}(t) \widetilde{\eta}_{j}\left(t^{\prime}\right)\right]=\delta_{i, j} \delta\left(t-t^{\prime}\right)$, so that the matrix $\widetilde{\boldsymbol{Q}}$ controls the covariance structure $^{20}$ of the noise. In this section, we leave the noise matrix $\widetilde{\boldsymbol{Q}}$ otherwise unspecified in order to derive general results. In chapter 3 , we consider two special cases of noise and their implications for the TWD and terrace width pair correlations.

In the limit of a sufficiently small $\varpi^{2}|\widetilde{\boldsymbol{Q}}|^{2}$, we treat the noise as a perturbation of (2.1). Because stable solutions of (2.1) respond linearly to small perturbations, we set $w_{i}(t)=\varpi\left[1+\xi_{i}(t)\right],\left|\xi_{i}\right| \ll 1$. By $(2.1)$ and (2.2), the linearized version of

\footnotetext{
${ }^{02}$ For a vector random variable, $\boldsymbol{X}$, the phrase "covariance structure" is used to mean the structure of the matrix $\mathbb{E}\left[X_{i} X_{j}\right]\left(X_{i}\right.$ : $i$ th component of $\left.\boldsymbol{X}\right)$. We consider whether this matrix: (i) is conservative (i.e., the sum of elements in any row or column is zero); (ii) is sparse; and/or (iii) has positive or negative off-diagonal elements. In this article, we consider $X_{i}=\varpi \widetilde{Q}_{i, j} \widetilde{\eta}_{j}$ and $X_{i}=\left(w_{i}-\varpi\right) / \varpi ;$ cf. $(2.4)$.
} 
(2.3) is

$$
\begin{aligned}
\dot{\xi}_{i}= & a F\left\{(1-p)\left(\xi_{i+1}-\xi_{i}\right)+p\left(\xi_{i}-\xi_{i-1}\right)+g\left[-(1+\beta)\left(\xi_{i+2}-3 \xi_{i+1}\right.\right.\right. \\
& \left.\left.\left.+3 \xi_{i}-\xi_{i-1}\right)+\beta\left(\xi_{i+1}-3 \xi_{i}+3 \xi_{i-1}-\xi_{i-2}\right)\right]\right\}+\widetilde{Q}_{i, j} \widetilde{\eta}_{j},
\end{aligned}
$$

where (abusing notation) we use the same symbol $\left(\xi_{i}\right)$ for the approximation of $\xi_{i}$, i.e., the solution of the linearized equations. The parameters $p, \beta$ and $g$ are defined by

$$
p=\frac{1}{2}\left(\frac{\nu}{\sinh ^{2} \nu}-\frac{e^{-\nu}}{\sinh \nu}\right), \quad \beta=\frac{1}{2} \frac{e^{-\nu}}{\sinh \nu}, \quad g=3 \frac{\breve{g} a^{2}}{T} \widetilde{m}_{0}^{3} \rho_{0},
$$

with $\nu=v \varpi /(2 D), \widetilde{m}_{0}=a / \varpi$ and $0<p<1 / 2$; cf. (34) in [34] where $g=0$. Note that $\xi_{i}=\left(w_{i}-\varpi\right) / \varpi$ is a stochastic process. We assume that fluctuations are small in probability: $1-\operatorname{Pr}\left[\sup _{t>0}\left|\xi_{i}(t)\right| \ll 1\right] \ll 1$ for all $i$ (Pr denotes the probability).

Let us pause for a moment and take a closer look at (2.4). For $g=0$ (no step interaction), this equation reduces to $\dot{w}_{i} \approx F a\left[(1-p)\left(w_{i+1}-w_{i}\right)+p\left(w_{i}-w_{i-1}\right)\right]$. We can consider $p$ as the fraction of deposited atoms that attach to the downstep of terrace $i$, in the setting of figure $2.1 ; 1-p$ is the fraction of atoms that move to an upstep. Hence, the number of atoms per unit time that cause an increase of the $i$ th terrace size is $p F\left(w_{i}-w_{i-1}\right)$ by competition with the upper terrace, and $(1-p) F\left(w_{i+1}-w_{i}\right)$ from the lower terrace. Here we ignore the possibility of nucleation. For a similar model, see [19] in regard to step motion in a diffusion bias (and [20] for an effect of impurities). 


\subsection{TWD and pair correlations: general formulas}

In this section, we solve SDEs (2.4) with particular emphasis on the pair correlation for terrace widths. Equation (2.4) is fully non-dimensionalized via $t \mapsto$ $\tau=t F a$ and $\widetilde{\eta}_{i}(t) \mapsto \eta_{i}(\tau)=(1 / \sqrt{F a}) \widetilde{\eta}_{i}(t), \widetilde{\boldsymbol{Q}} \mapsto \boldsymbol{Q}=(1 / \sqrt{F a}) \widetilde{\boldsymbol{Q}}$. SDEs (2.4) are then recast to the matrix form

$$
\frac{\mathrm{d} \boldsymbol{\xi}}{\mathrm{d} \tau}=-\boldsymbol{A} \cdot \boldsymbol{\xi}+\boldsymbol{Q} \cdot \boldsymbol{\eta}
$$

where $\boldsymbol{\xi}=\left(\xi_{0}, \ldots, \xi_{N-1}\right), \boldsymbol{\eta}=\left(\eta_{0}, \ldots, \eta_{N-1}\right)$, and $\boldsymbol{A}$ is a sparse circulant matrix with first-row entries $[1-2 p+3 g(1+2 \beta),-1+p-g(3+4 \beta), g(1+\beta), 0, \ldots, 0, g \Re, p-$ $g(1+43)]$. The (non-dimensional) matrix $\boldsymbol{Q}$ is only required to be circulant and constant.

By integrating (2.6), we obtain

$$
\boldsymbol{\xi}(t)=\int_{0}^{\tau} e^{-\boldsymbol{A}(\tau-s)} \boldsymbol{Q} \cdot \boldsymbol{\eta}(s) \mathrm{d} s, \quad \tau=t F a
$$

Evidently, for every $t>0,(2.7)$ describes a vector Gaussian random variable with zero expectation. The joint probability density of any number of terrace widths is Gaussian. In light of the initial condition $\boldsymbol{\xi}(0)=0$, the covariance matrix is circulant, given by the expression (see A)

$$
\begin{array}{r}
\mathfrak{C}_{m}(t(\tau) ; N):=N^{-1} \mathbb{E}\left[\xi_{j}(t) \xi_{j+m}(t)\right]= \\
\frac{1}{N} \int_{0}^{\tau} \mathrm{d} s \int_{0}^{\tau} \mathrm{d} s^{\prime} \mathbb{E}\left\{\eta_{j}\left(s^{\prime}\right)\left[e^{-\boldsymbol{A}^{T}\left(\tau-s^{\prime}\right)}\right]_{j, k} Q_{k, l}^{T} Q_{l, n}\left[e^{-\boldsymbol{A}(\tau-s)}\right]_{n, p}\left[\eta(s)_{m}^{c}\right]_{p}\right\}
\end{array}
$$

where $\left[\eta(s)_{m}^{c}\right]$ is the $m$ th cyclic permutation of the vector $\boldsymbol{\eta},{ }^{30}$ and the symbol $\boldsymbol{A}^{T}$

${ }^{03}$ Note that ODEs (2.4) under the initial condition $\boldsymbol{\xi}(0)=0$ yield $j$-independent covariances, $\mathfrak{C}_{m}$ 
denotes the transpose of $\boldsymbol{A}$. Recall that $\mathbb{E}\left[\eta_{j}(s) \eta_{k}\left(s^{\prime}\right)\right]=\delta_{j, k} \delta\left(s-s^{\prime}\right)$. In the case with $m=0,(2.8)$ reduces to a formula for the terrace width variance, viz.,

$$
\mathfrak{C}_{0}(t ; N)=\mathbb{E}\left[\xi_{j}^{2}(t)\right]=N^{-1} \mathbb{E}\left[\boldsymbol{\xi}(t)^{2}\right]=\int_{0}^{t F a}\left|e^{-\boldsymbol{A} s} \boldsymbol{Q}\right|^{2} \mathrm{~d} s .
$$

The probability density for a single (scaled) terrace width, $\xi_{i}$, is ${ }^{40}$

$$
P(\xi, t ; N)=\frac{1}{\sqrt{2 \pi \mathfrak{C}_{0}(t ; N)}} \exp \left[-\frac{\xi^{2}}{2 \mathfrak{C}_{0}(t ; N)}\right], \quad-\infty<\xi<\infty,
$$

by which the TWD (for the dimensional terrace width, $w_{i}$ ) is obtained via $\xi=$ $(w-\varpi) / \varpi,-\infty<w<\infty$. Within this approximation, $\operatorname{Pr}\left[w_{i}<0\right]>0$ : there exists a nonzero probability of step crossing. This likelihood can be controlled by the step interaction strength, deposition rate and initial terrace width (see section 4.4). We henceforth consider $\operatorname{Pr}\left[w_{i}<0\right]$ as negligibly small.

\subsection{Covariance matrix: large- $N$ limit}

Next, we compute $\mathfrak{C}_{m}$ by (2.8). First, we derive an equivalent expression valid for finite $N$ and $t$. Second, we enforce the limit $N \rightarrow \infty$ and thereby extract a single-integral formula for $\mathfrak{C}_{m}(t):=\mathfrak{C}_{m}(t ; \infty)$. We then simplify this formula by taking $\mathcal{O}(N)>t F a \gg 1: t F a$ is treated as large yet independent of $N$.

By a property of circulant matrices (see A), we write (2.8) as

$$
\begin{array}{r}
\mathfrak{C}_{m}(t ; N)=\int_{0}^{\tau} N^{-1} \sum_{k=0}^{N-1} \vartheta_{k} e^{-\lambda_{k} s} \cos \left(\frac{2 \pi k m}{N}\right) \mathrm{d} s \\
=N^{-1} \sum_{k=0}^{N-1} \vartheta_{k} \lambda_{k}^{-1}\left(1-e^{-\lambda_{k} \tau}\right) \cos \left(\frac{2 \pi k m}{N}\right),
\end{array}
$$

\footnotetext{
${ }^{04}$ By abusing notation, we use the same symbol, $\xi$, to denote both the independent real variable of the TWD and the stochastic process associated with each terrace width.
} 
where $\lambda_{k}$ and $\vartheta_{k}$ are the eigenvalues of $\boldsymbol{A}+\boldsymbol{A}^{T}$ and $\boldsymbol{Q} \boldsymbol{Q}^{T}$, respectively. The eigenvalues of (square) circulant matrices can be evaluated directly via the discrete Fourier transform [41]. Since both $\boldsymbol{A}$ and $\boldsymbol{Q}$ are circulant, $\boldsymbol{A}+\boldsymbol{A}^{T}$ and $\boldsymbol{Q} \boldsymbol{Q}^{T}$ are both circulant and symmetric. A simple calculation reveals that $\lambda_{k}$ and $\vartheta_{k}$ share the same general form: they are given by ${ }^{50}$

$$
\lambda_{k}=r_{0}+2 \sum_{j=1}^{M} r_{j} \cos \left(\frac{2 \pi j k}{N}\right), \quad \vartheta_{k}=s_{0}+2 \sum_{j=1}^{M} s_{j} \cos \left(\frac{2 \pi j k}{N}\right)
$$

The coefficients $r_{j}$ and $s_{j}$ depend on the precise forms of $\boldsymbol{A}$ and $\boldsymbol{Q}$, respectively. We specify these coefficients later. In (2.12), we define $M=(N-1) / 2$ for odd $N$, and $M=(N-2) / 2$ for even $N$.

By taking the limit $N \rightarrow \infty$, the sum in (2.11) becomes an integral:

$$
\mathfrak{C}_{m}(t)=\mathfrak{C}_{m}(t ; \infty)=\int_{0}^{1} \mathrm{~d} y \vartheta(y) \lambda(y)^{-1}\left[1-e^{-\lambda(y) \tau}\right] \cos (2 \pi m y)
$$

where $\vartheta(y)$ and $\lambda(y)$ are the limits of $\vartheta_{k}$ and $\lambda_{k}$ as $N \rightarrow \infty$, and $y$ is the continuous variable replacing $k / N ; 0<y<1$. The variance of the TWD is found by setting $m=0$ in $(2.13)$, which readily produces the result given in [35].

We comment on the interpretation of $\lambda(y)$ and $\vartheta(y)$. When $N$ is finite, the sequences $\left\{\lambda_{k}\right\}$ and $\left\{\vartheta_{k}\right\}$ contain information about nearest-neighbor interactions and noise correlations, respectively. In particular, each nonzero coefficient $r_{j}\left(s_{j}\right)$ in (2.12) arises from the existence of interactions (noise correlations) between a given

\footnotetext{
05 This property follows directly from the method of calculating the eigenvalues of a circulant matrix. Specifically, if $\mathcal{C}$ is circulant, its eigenvalues $c_{k}$ are given by $c_{k}=F_{k, l} \mathcal{C}_{l, 0}$, where $\boldsymbol{F}$ is the discrete Fourier transform. Note that $F_{j, k}=\exp (-2 \pi i j k / N)$, where $i^{2}=-1$. If, in addition, $\mathcal{C}$ is symmetric, the formula for $c_{k}$ (where $c_{k}$ is $\lambda_{k}$ or $\vartheta_{k}$ ) reduces to $(2.12)$.
} 
terrace and its $j$ th nearest neighbor on each side. In the limit $N \rightarrow \infty$, formulas (2.12) become continuous Fourier decompositions and $k / N \rightarrow y$ measures the surface height difference between two terraces. The continuous functions $\lambda(y)$ and $\vartheta(y)$ can yield information about the range of interactions and noise correlations as measured by the continuous variable $y$. If the largest index $j^{\prime}$ of the nonzero coefficients $r_{j}$ (or $s_{j}$ ) becomes $j^{\prime}=\mathcal{O}(N)$, the interactions (or noise correlations) extend to finite, $\mathcal{O}(1)$ values of $y$ as $N \rightarrow \infty .^{60}$

In chapter 3 , we consider two cases in which the maximum index $j^{\prime}$ appearing in (2.12) is fixed and $\mathcal{O}(1)$ in the limit of large $N$; interactions and noise correlations are thus restricted to a few nearest neighbors for a given terrace site. This assumption leads to terrace width pair correlations that decay exponentially with respect to the nearest-neighbor index $m: \mathfrak{C}_{m} \propto c^{|m|}, 0<c<1$ (where $c$ is defined in chapter 3).

\footnotetext{
${ }^{06}$ In the discrete setting (with finite $N$ ), the $j$ th nearest neighbor is identified with the terrace that is $j$ atomic heights below (or above) a given terrace. Consequently, the $j$ th nearest neighbor amounts to a height difference given by the limiting value $j a$ as $a \downarrow 0$, with $N a$ kept fixed. If $j=\mathcal{O}(N)$, the limiting value of $j a$ corresponds to a nonzero, finite height difference along the step train. If $j=\mathcal{O}(1)$, the limiting value of $j a$ corresponds to a practically zero height difference.
} 


\section{Chapter 3}

\section{Particular cases of noise}

In this section, we find the terrace width covariance matrix for two different forms of the noise matrix $\boldsymbol{Q}$. This procedure is motivated by the observation that the origins of noise should in principle depend on whether or not there is significant deposition.

In section 3.1, we introduce noise at the level of adatom diffusion, assuming that the system is sufficiently close to equilibrium. The noise coefficients are determined by the fluctuation-dissipation theorem [9]. By solving the free-boundary problem for step motion, we then obtain a second-order conservative noise for the motion of terraces. In section 3.2, we consider an asymmetric, first-order conservative noise whose amplitude depends on the deposition flux $(F)$, based on a proposal in [10]: the noise coefficient is chosen to reflect (phenomenologically) the asymmetric attachment of atoms at step edges in correspondence to the drift velocity, $v=F a \varpi$. Loosely speaking, this latter noise should apply to a system far enough from equilibrium so that near-equilibrium statistics do not hold for adatoms.

\subsection{Adatom noise under fluctuation-dissipation theorem}

In this section, we derive $\boldsymbol{Q}$ by adding noise to the adatom equations of motion and solving the free boundary problem for terraces. Let us begin with a modified 
diffusion equation for adatoms in the step comoving frame, $\left(D \partial_{x}^{2}+v \partial_{x}\right) \varrho_{i}(x)+F-$ $\partial_{x} q(x, t)=0$ for terrace $i$, where we applied $\partial_{t} \varrho_{i} \approx 0$ (quasistatic approximation). The noise source $q(x, t)$ is delta-correlated in time and space, and contributes to a random adatom flux [9]. Since we consider a (continuous) adatom density in the BCF model [11], $q$ is intended to capture the effect of microscopic motions, which are usually left out of a deterministic continuum picture for terraces. The $x$-derivative acting on $q$ ensures that the resulting noise is conservative (consistent with Fick's law for adatom diffusion).

The diffusion equation is supplemented with corresponding, modified boundary conditions: $-J_{i}\left(x_{i}\right)=\kappa\left[\varrho_{i}\left(x_{i}\right)-\varrho_{i}^{\mathrm{eq}}+\varsigma_{i}^{+}\right]$and $J_{i}\left(x_{i+1}\right)=\kappa\left[\varrho_{i}\left(x_{i+1}\right)-\varrho_{i+1}^{\mathrm{eq}}+\varsigma_{i+1}^{-}\right]$, where $J_{i}(x)=-D \partial_{x} \varrho_{i}-v \varrho_{i}+q(x)$ is the modified adatom flux on the $i$ th terrace. The terms $\varsigma_{i}^{ \pm}$are Langevin forces accounting for random attachment and detachment events on the right $(+)$ and left $(-)$ sides of the $i$ th step edge [9]. The terms $\varrho_{i}^{\mathrm{eq}}$ and $\kappa$ are discussed in section 2.1 .

In order to determine the covariance structure of $\varsigma_{i}^{ \pm}$and $q$, we follow [9] in applying the fluctuation-dissipation theorem. First, suppose the system is in equilibrium (i.e., with $F=0$, since we neglect desorption), where

$$
\begin{array}{r}
\mathbb{E}\left[q(x, t) q\left(x^{\prime}, t^{\prime}\right)\right]=2 D \rho_{0} \delta\left(x-x^{\prime}\right) \delta\left(t-t^{\prime}\right), \\
\mathbb{E}\left[\varsigma_{i}^{\alpha}(t) \varsigma_{j}^{\beta}\left(t^{\prime}\right)\right]=\left(2 \rho_{0} / \kappa\right) \delta_{i, j} \delta\left(t-t^{\prime}\right) \delta_{\alpha, \beta}, \quad \alpha, \beta= \pm .
\end{array}
$$

If the system is sufficiently close to equilibrium, one may replace $\rho_{0}$ by the average of $\varrho_{i}(x)$. To simplify the analysis, we assume that corrections arising from this substitution are negligible, and henceforth consider only the noise defined by (3.1) 
and (3.2).

By solving the diffusion equation in the limit of DL kinetics, one can find linearized SDEs for terrace motion that have the same form as (2.4), with the exception of the noise term. This term is now modified according to the replacement

$$
\widetilde{Q}_{i, j} \widetilde{\eta}_{j} \rightarrow 2 M_{i}\left(w_{i}\right)-M_{i-1}\left(w_{i-1}\right)-M_{i+1}\left(w_{i+1}\right)
$$

where

$$
M_{i}\left(w_{i}, t\right)=\frac{\tilde{m}_{0} v e^{v w_{i} / 2 D}}{2 D \sinh \left(v w_{i} / 2 D\right)} \int_{x_{i}}^{x_{i+1}} \mathrm{~d} z e^{-v\left(x_{i+1}-z\right) / D} q(z, t)
$$

In the DL case, contributions from $\varsigma^{ \pm}$do not appear in (3.4) since the DL limit, where $\kappa \rightarrow \infty$, implies $\mathbb{E}\left[\varsigma_{i}^{ \pm} \varsigma_{j}^{ \pm}\right] \rightarrow 0$ (cf. (3.2)).

In the small fluctuation limit, we approximate $w_{i} \approx \varpi$ in the definition of $M_{i}\left(w_{i}, t\right) \cdot{ }^{70}$ Thus, the $M_{i}\left(w_{i}, t\right) \approx M_{i}(\varpi, t)$ can be treated as independent Gaussian random variables whose variances depend on the average terrace width. The noise is reduced to the form given in (2.4):

$$
\widetilde{Q}_{i, j} \widetilde{\eta}_{j}=2 M_{i}-M_{i-1}-M_{i+1}
$$

where these $M_{i}$ are independent Gaussian random variables satisfying

$$
\mathbb{E}\left[M_{i} M_{j}\right]=\frac{\widetilde{m}_{0}^{2} v \rho_{0} \sinh (2 \nu)}{2(\sinh \nu)^{2}} \delta_{i, j} \delta\left(t-t^{\prime}\right)
$$

After some algebra, we obtain formulas for the eigenvalues of the matrices

${ }^{07}$ A formal argument for this approximation can be made by adding to ODEs (2.1) the "small" noise $\epsilon\left[2 M_{i}\left(w_{i}\right)-M_{i-1}\left(w_{i-1}\right)-M_{i+1}\left(w_{i+1}\right)\right]$ where $0<\epsilon \ll 1$. Then, use the expansion $w_{i}(t)=$ $\varpi\left[1+\epsilon \xi_{i}(t)+\ldots\right]$. Making the substitution $w_{i}, w_{i \pm 1} \rightarrow \varpi$ amounts to keeping only the leadingorder terms in the resulting $\epsilon$-expansion of the SDEs. 
$\boldsymbol{A}+\boldsymbol{A}^{T}$ and $\boldsymbol{Q} \boldsymbol{Q}^{T}$ (defined in sections 2.2 and 2.3):

$$
\begin{gathered}
\lambda_{k}=2\left[1-2 p+2 g(1+2 \beta)\left(1-\cos \frac{2 \pi k}{N}\right)\right]\left(1-\cos \frac{2 \pi k}{N}\right), \\
\vartheta_{k}=\frac{2 \widetilde{m}_{0}^{2} \varpi \rho_{0} \sinh (2 \nu)}{(\sinh \nu)^{2}}\left(1-\cos \frac{2 \pi k}{N}\right)^{2} .
\end{gathered}
$$

With these definitions of $\vartheta_{k}$ and $\lambda_{k}$, we apply (2.13) to calculate the covariance matrix (for $|m|=0,1, \ldots$ ):

$$
\begin{aligned}
\mathfrak{C}_{m}(t)= & \frac{\widetilde{m}_{0}^{2} \varpi \rho_{0} \sinh (2 \nu)}{(\sinh \nu)^{2}} \int_{0}^{1} \mathrm{~d} y \frac{[1-\cos (2 \pi y)] \cos (2 \pi m y)}{(1-2 p)+2 g(1+2 \beta)(1-\cos (2 \pi y))} \\
& \times\left\{1-e^{-\tau[2(1-2 p)+4 g(1+2 \beta)(1-\cos (2 \pi y))(1-\cos (2 \pi y))]}\right\} .
\end{aligned}
$$

We have not been able to compute this $\mathfrak{C}_{m}(t)$ in simple closed form for finite $t$. In the limit $t \rightarrow \infty$, the exponential term in the integrand of (3.9) can be dropped (see A.1); thus, we obtain the steady-state formulas

$$
\begin{array}{r}
\mathfrak{C}_{0, \mathrm{st}}=\frac{\widetilde{m}_{0}^{2} \varpi \rho_{0} \sinh (2 \nu) /(\sinh \nu)^{2}}{[(1-2 p)+2 g(1+2 \beta)]} \frac{\alpha+\sqrt{1-\alpha^{2}}-1}{\alpha \sqrt{1-\alpha^{2}}}, \\
\mathfrak{C}_{m, \mathrm{st}}=-\frac{\widetilde{m}_{0}^{2} \varpi \rho_{0} \sinh (2 \nu) /(\sinh \nu)^{2}}{(1-2 p)+2 g(1+2 \beta)} \frac{1-\alpha}{\alpha \sqrt{1-\alpha^{2}}}\left[\frac{1-\sqrt{1-\alpha^{2}}}{\alpha}\right]^{|m|},
\end{array}
$$

where $|m| \geq 1$ and $\alpha=2 g(1+2 \beta) /[(1-2 p)+2 g(1+2 \beta)](0<\alpha<1)$. Evidently, covariances decay exponentially with $m$.

Remark 1. The terrace width correlations given by (3.11) are all negative. This means that $\operatorname{sgn}\left(\xi_{i}\right)=-\operatorname{sgn}\left(\xi_{i \pm 1}\right)$ with probability greater than $1 / 2$, where $\operatorname{sgn}(x)=$ $x /|x|$ for $x \neq 0$. Thus, short terraces are more likely to be surrounded by long terraces, and vice versa. Hence, the noise of (3.5) disfavors the formation of step bunches. 
Remark 2. If $F \downarrow 0$ (i.e., $\nu=F a \varpi^{2} /(2 D) \downarrow 0$, or $p \uparrow 1 / 2$ ) and $g=\mathcal{O}(1)>0$, by (3.10) the steady-state variance approaches a nonzero value: $\mathfrak{C}_{0, \mathrm{st}} \rightarrow \widetilde{m}_{0}^{2} \varpi \rho_{0} / g$; see figure 3.1 recalling that, by $(2.5), \beta=\beta(p)$ through $\nu$. By (3.11), off-diagonal elements of the steady-state covariance (circulant) matrix approach zero; that is, $\mathfrak{C}_{m, \mathrm{st}} \rightarrow 0$ as $F \downarrow 0$ for $m \neq 0$. The above result for $\mathfrak{C}_{0, \mathrm{st}}$ is in agreement with the long-time limit of the TWD found in [35], where a similar model is invoked without deposition flux (for $F=0$ ). In the limit $F \rightarrow \infty$, by (3.10) and (3.11) we compute

$$
\begin{array}{r}
\left.\mathfrak{C}_{0, \mathrm{st}}\right|_{F \rightarrow \infty}=\frac{2 \widetilde{m}_{0}^{2} \varpi \rho_{0}}{1+2 g}\left[\frac{\alpha_{*}+\sqrt{1-\alpha_{*}^{2}}-1}{\alpha_{*} \sqrt{1-\alpha_{*}^{2}}}\right], \\
\left.\mathfrak{C}_{m, \mathrm{st}}\right|_{F \rightarrow \infty}=\frac{-2 \widetilde{m}_{0}^{2} \varpi \rho_{0}}{1+2 g}\left[\frac{1-\alpha_{*}}{\alpha_{*} \sqrt{1-\alpha_{*}^{2}}}\right]\left[\frac{1-\sqrt{1-\alpha_{*}^{2}}}{\alpha_{*}}\right]^{|m|},|m| \geq 1,
\end{array}
$$

where $\alpha_{*}=\lim _{F \rightarrow \infty} \alpha(F)=2 g /(1+2 g)$; cf. figure 3.1 .

\subsection{Deposition-flux-induced noise at steps}

In this section, we consider a phenomenological noise that arises solely as a result of deposition, by choosing not to apply the fluctuation-dissipation theorem. Our approach is partly motivated by the fact that deposition is a non-equilibrium process. We propose a noise motivated by inspection of the linearized deterministic equations for $g=0$, following the phenomenological model of [10]. The idea is to consider the number of atoms arriving at each step edge as fluctuating in accord with the $p$-induced asymmetry, i.e., allow lateral fluxes that cause random noise to distinguish downsteps from upsteps.

Let $\mathcal{N}_{i}^{ \pm}(t)$ be the (random) number of atoms attaching to an upstep $(+)$ or 


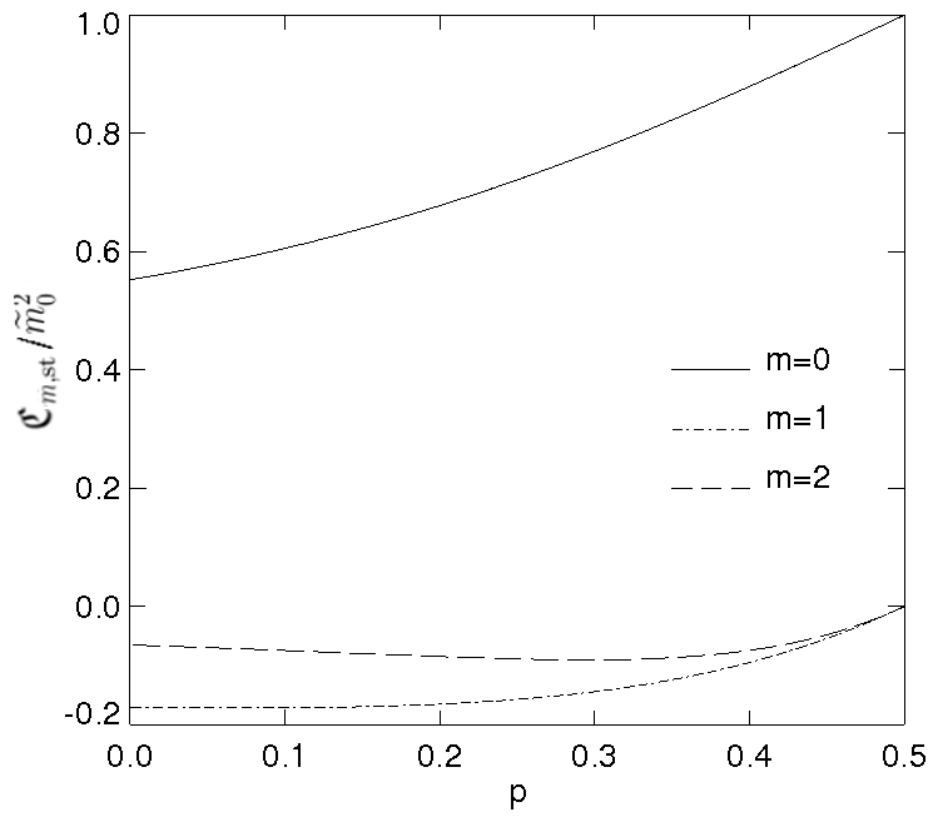

Figure 3.1: Steady-state, scaled covariance matrix, $\mathfrak{C}_{m, \mathrm{st}} / \widetilde{m}_{0}^{2}$, as function of $p$ for $m=0$ (variance) and $m=1,2$ (covariance for first and second nearest neighbor). We employ $\varpi \varrho_{o}=g=1$. 
a downstep (-) of the $i$ th terrace. For macroscopic times and each $i$, we posit that the increments $\mathcal{N}_{i}^{ \pm}\left(t_{n+1}\right)-\mathcal{N}_{i}^{ \pm}\left(t_{n}\right)$ are independent, normally distributed and stationary random variables, with mean zero and variance proportional to $1-p(+)$ or $p(-)$ times $\left(t_{n+1}-t_{n}\right) F w_{i}$, where $0<t_{n}<t_{n+1}$.

Accordingly, we perturb (2.4), or (2.1), for the $i$ th terrace motion by: (i) the noise $\sqrt{(1-p) F w_{i+1} \Omega} \widetilde{\eta}_{i+1}$ (which has the dimension of speed) for fluctuations in the number of atoms attaching to the upstep bounding the $(i+1)$-th terrace; (ii) $\sqrt{p F w_{i-1} \Omega} \widetilde{\eta}_{i-1}$ regarding the downstep of the $(i-1)$ th terrace; and (iii) $\left[\sqrt{p F w_{i} \Omega}-\right.$ $\left.\sqrt{(1-p) F w_{i} \Omega}\right] \widetilde{\eta}_{i}$ for mass conservation purposes. See also the discussion following (2.4) and (2.5) for additional motivation. (Recall that $\left(\widetilde{\eta}_{0}, \ldots, \widetilde{\eta}_{N-1}\right)$ is a vector white noise, having independent, identically distributed components $\left.\widetilde{\eta}_{i}=\mathrm{d} \mathcal{B}_{i} / \mathrm{d} t\right)$. We now propose the noise $\operatorname{model}^{8}$

$$
\widetilde{Q}_{i, j} \widetilde{\eta}_{j}=\frac{a}{\varpi} \sqrt{F \varpi}\left[\sqrt{1-p}\left(\widetilde{\eta}_{i+1}-\widetilde{\eta}_{i}\right)+\sqrt{p}\left(\widetilde{\eta}_{i}-\widetilde{\eta}_{i-1}\right)\right] .
$$

0 .

With this choice, the eigenvalues of the noise matrix $\widetilde{\boldsymbol{Q}} \widetilde{\mathbf{Q}}^{T}=(1 / F a) \boldsymbol{Q} \boldsymbol{Q}^{T}$ are

$$
\vartheta_{k}=2 \widetilde{m}_{0}\left[1+2 \sqrt{p(1-p)} \cos \frac{2 \pi k}{N}\right]\left(1-\cos \frac{2 \pi k}{N}\right) .
$$

${ }^{08}$ In (3.14), notice the replacement of $w_{i}$ and $w_{i \pm 1}$ by the initial terrace width, $\varpi$, in the noise diffusion coefficients. A formal argument for this approximation can be made by adding to ODEs (2.1) the "small" term $\epsilon \widetilde{\eta}_{i} \sqrt{F w_{i} a^{2}}(\sqrt{p}-\sqrt{1-p})+\epsilon \widetilde{\eta}_{i+1} \sqrt{1-p} \sqrt{F w_{i+1} a^{2}}-\epsilon \widetilde{\eta}_{i-1} \sqrt{p} \sqrt{F w_{i-1} a^{2}}$ where $0<\epsilon \ll 1$. Then, (3.14) are viewed as the lowest-order equations in $\epsilon$. 
Accordingly, using (2.13), we derive the formula (for $m=0, \pm 1, \pm 2, \ldots$ )

$$
\begin{aligned}
\mathfrak{C}_{m}(t) & =\int_{0}^{1} \mathrm{~d} y \frac{\widetilde{m}_{0} \cos (2 \pi m y)[1+2 \sqrt{p(1-p)} \cos (2 \pi y)]}{(1-2 p)+2 g(1+2 \beta)[1-\cos (2 \pi y)]} \\
& \times\left\{1-e^{-\tau[2(1-2 p)+4 g(1+2 \beta)(1-\cos (2 \pi y))(1-\cos (2 \pi y))]}\right\} .
\end{aligned}
$$

As $t F a \rightarrow \infty$ with $g, \nu=\mathcal{O}(1)>0$, the exponential term can be dropped; thus, the steady-state covariance matrix is comprised of the elements (see A.1)

$$
\begin{aligned}
\mathfrak{C}_{0, \mathrm{st}} & =\frac{\widetilde{m}_{0}}{2 g(1+2 \beta) \sqrt{1-\alpha^{2}}}\left[\alpha+2 \sqrt{p(1-p)}\left(1-\sqrt{1-\alpha^{2}}\right)\right], \\
\mathfrak{C}_{m, \mathrm{st}} & =\frac{\widetilde{m}_{0}}{2 g(1+2 \beta) \sqrt{1-\alpha^{2}}}[\alpha+2 \sqrt{p(1-p)}]\left[\frac{1-\sqrt{1-\alpha^{2}}}{\alpha}\right]^{|m|},
\end{aligned}
$$

where $|m| \geq 1$ and $\alpha=2 g(1+2 \beta) /[(1-2 p)+2 g(1+2 \beta)]$; see figure 3.2 .

We note in passing that, in the special (idealized) limit $g \downarrow 0$ (vanishing step interaction), the time-dependent TWD variance becomes (see A.1)

$$
\left.\mathfrak{C}_{0}(t)\right|_{g \downarrow 0}=\frac{\widetilde{m}_{0}}{1-2 p}\left\{1-e^{-\breve{t}}\left[I_{0}(\breve{t})+2 \sqrt{p(1-p)} I_{1}(\breve{t})\right]\right\}
$$

where $\breve{t}=2(1-2 p) t F a$ and $I_{n}(t)$ is the modified Bessel function of $n$th order [42]. In the limit $\breve{t} \rightarrow \infty$, we obtain $\left.\mathfrak{C}_{0}(t)\right|_{g \downarrow 0} \rightarrow \widetilde{m}_{0}(1-2 p)^{-1}$.

Remark 3. If $\nu=F a \varpi^{2} /(2 D) \downarrow 0$ (i.e., $p \uparrow 1 / 2$ ) and $g=\mathcal{O}(1)>0$, by (3.15) the covariance approaches zero for any time $t$ and all $m$. This behavior is derived by use of $\beta=\mathcal{O}\left(\nu^{-1}\right)$ as $\nu \downarrow 0$, and is consistent with SDEs (2.4), since these equations become (deterministic) ODEs in view of (3.14) as $F \downarrow 0$. Details for the precise role of time $t$ in this limiting case are provided in Remark 4 and A.4; see also figure 3.3 for $m=0$. 


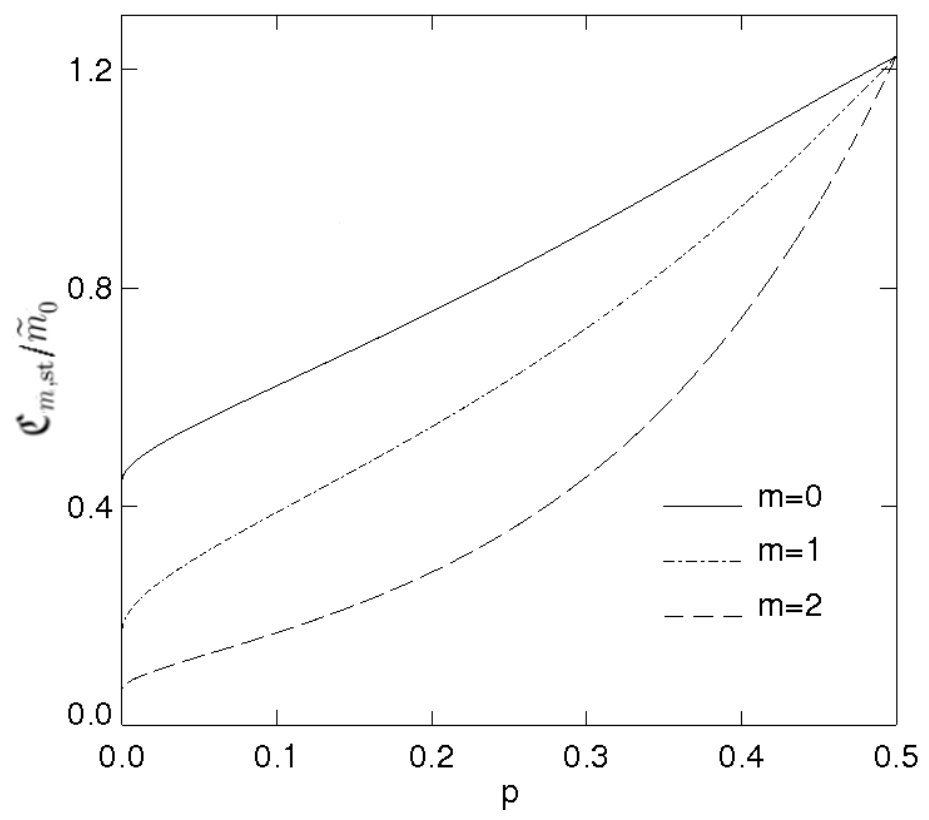

Figure 3.2: Steady-state, scaled covariance matrix, $\mathfrak{C}_{m, \text { st }} / \widetilde{m}_{0}$, as function of $p$ for $m=0$ (variance) and $m=1,2$ (covariance for first and second nearest neighbor).

We use $g=1$. The narrowing of the TWD with $F$ (decreasing $p$ ) is evident. 


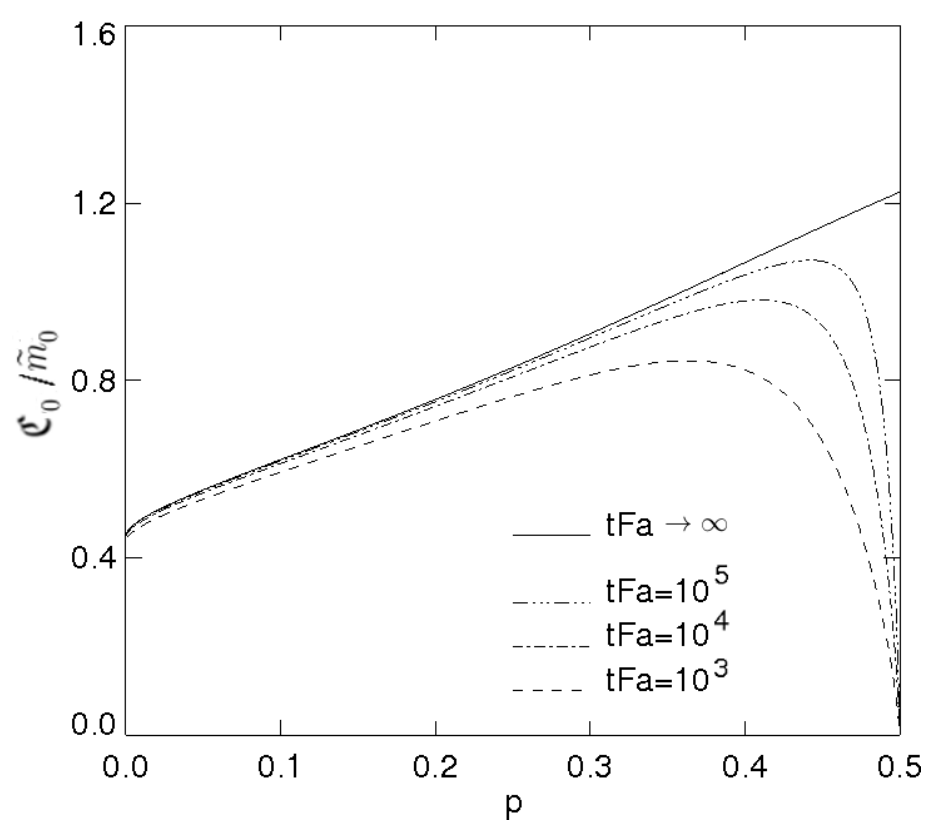

Figure 3.3: Time-dependent, scaled TWD variance, $\mathfrak{C}_{0}(t) / \widetilde{m}_{0}$, computed by numerical integration of (3.15) (dashed curves) versus the steady-state variance of (3.16) (solid line); $g=1$. For large $t F a$, the time-dependent variance converges to its steady-state value non-uniformly in $p$ : the depicted behavior near $p=1 / 2$ indicates that the limits $F \downarrow 0$ and $t \rightarrow \infty$ do not commute (cf. Remark 4). 
Remark 4. Interestingly, the limits $\nu \downarrow 0$ and $t \rightarrow \infty$ of (3.15) do not commute (for any $m$ ): the steady-state values (3.16) and (3.17) approach the finite value $\sqrt{3 \widetilde{m}_{0}^{2} / 2 g}$ as $\nu \downarrow 0$, whereas, by $(3.15), \mathfrak{C}_{m}(t) \rightarrow 0$ as $p \uparrow 1 / 2$ for fixed $t>0$ and $g$ (cf. figures 3.2, 3.3 and Remark 3). To resolve this apparent paradox, we point out a transition in the asymptotics for $\mathfrak{C}_{m}$ if $t F a=\mathcal{O}\left(g \nu^{-3}\right)$. If $\nu$ is small, the $\mathfrak{C}_{m}$ given by (3.16) and (3.17) is recovered from (3.15) if aFt is large enough so that the vanishing exponential term does not suppress the emerging singularity in the integrand of (3.15). More precisely, for $\nu a F t \gg 1$ the major contribution to integration comes from the vicinity of $y=0$. Thus, to extract the steady-state limits (3.16) and (3.17) we require that $1-2 p>\mathcal{O}\left[g(1+2 \beta) y^{2}\right]$ and $(1-2 p) y^{2} a F t=\mathcal{O}(1)$, by which $t F a \gg g \nu^{-3}$. By contrast, if $a F t \ll g \nu^{-3}$, then $\mathfrak{C}_{m}(t)$ can be arbitrarily close to zero (see A.4 for further technical details).

Remark 5. Consider the steady-state variance $\mathfrak{C}_{0, \mathrm{st}}(p, g)$ of $(3.16)$ for $1-2 p>0$. For fixed $p, \mathfrak{C}_{0, \text { st }}$ decreases with $g$. For finite and fixed $g, \mathfrak{C}_{0, \text { st }}$ increases with $p$, and thus decreases with $F$. The parameter $\beta$ decreases with $F$ and becomes exponentially small (compared to unity) if $\nu=v \varpi /(2 D) \gg 1$. Therefore, (3.17) predicts a narrowing of the TWD with increasing step interaction or deposition rate (see section 4.3).

Remark 6. The correlations given by (3.17) are positive, in contrast to the correlations associated with the adatom noise of section 3.1 ; cf. (3.11). In the steady state, this means that $\operatorname{sgn}\left(\xi_{i}\right)=\operatorname{sgn}\left(\xi_{i \pm 1}\right)$ with probability greater than $1 / 2$. Therefore, the noise given by (3.14) gives rise to a stepped surface in which long trains of wide 
terraces are likely to be followed by step bunches. We emphasize that the structure of terrace width correlations does not come specifically from the flux dependence of the noise, but rather from the generic, asymmetric form of $\boldsymbol{Q}$ introduced by (3.14). 


\section{Chapter 4}

\section{Discussion}

In this section, we discuss the results of chapters 2 and 3 , with particular emphasis on the relationship between noise structure and terrace width correlations. We also compare the result of section 3.2 to a mean field approach [16,34], outline a plausible connection to experiments, and discuss the validity of our linearized model.

\subsection{Noise structure and correlations}

A major component of our work has been to determine the terrace width covariance structures that arise from specific choices of conservative noise (cf. (2.13), (3.11), and (3.17)). For systems in equilibrium, the fluctuation-dissipation theorem allows one to calculate the structure of the noise in a system from known correlations of thermodynamic variables, and vice versa. We emphasize that application of this theorem is contingent upon knowledge of the steady state of the system, which acts as a constraint in determining the noise.

An alternate perspective, which we adopt here, is that the noise should be constrained by geometric properties of the system. First, the total system size should not fluctuate. This requirement is satisfied by choosing the noise to be conservative; that is, the sum of elements in any row or column of $\boldsymbol{Q}$ must be zero. This choice implies that summing equations (2.3) over all $i$ yields the deterministic equation 
$\sum_{i} \dot{\xi}_{i}=0$, so that the total length of the system is constant. As an additional constraint, we require the covariance matrix to be bounded for long times. This requirement must be checked for each $\boldsymbol{Q}$ by taking the limit $t \rightarrow \infty$ in $(2.13) .{ }^{90}$

For the two cases examined in sections 3.1 and 3.2, we find that our geometric constraints are sufficient to force the system to a steady state having a well-defined covariance matrix. Hence, our choices of noise are consistent with the fluctuationdissipation theorem, insofar as there exist steady-state covariances that in turn imply our noises by the fluctuation-dissipation theorem. Such an application of the fluctuation-dissipation theorem does not require the system to attain or be near equilibrium.

The study of terrace width fluctuations in the BCF framework with noise provides a means of predicting how terrace width correlations should behave in a continuum description of surfaces. If we take $a=\mathcal{O}\left(N^{-1}\right)$ as $N \rightarrow \infty$, then we can view the surface in terms of a continuous height profile (see section 2.4). The discrete solution, $\xi_{i}$, for terrace widths is mapped to a continuous variable, $\xi(h)$ : $\xi_{i} \mapsto \xi(h)$; this $\xi(h)$ measures the relative deviation of the slope from its average value at height $h$ (cf. the discussion following (2.13), especially the footnote). In our formulation, $h=y$. Because the covariance matrices (3.11) and (3.17) depend on $m=h / a$, we see that values of $\xi(h)$ separated by finite $h$ are not correlated.

\footnotetext{
09 The reader should not infer that the fixed system size constraint necessarily implies a bounded TWD variance; see [35] for an (unphysical) counter-example of a conservative noise yielding a TWD variance that is unbounded for long times. For finite (but large) $N$, this behavior [35] means that the TWD variance tends to become of the order of the system size at long times.
} 
This conclusion holds despite key differences in the structure and effects of the noise models used in sections 3.1 and 3.2. Specifically, the noise (3.3) results in terrace widths that are anti-correlated (no step bunching), while (3.14) leads to correlated terrace widths (step bunching). However, these detailed correlations are "washed out" when $m a$ is a macroscopic length (comparable to the size of the system), so that $|m| \gg 1$. This limit corresponds to a coarse-graining of the stepped surface.

\subsection{Mean field approach and decorrelation hypothesis}

Heuristically speaking, the main goal of a mean field formalism is to reduce the SDE system to a single Langevin-type equation that produces the same TWD as the starting, coupled system $[34,35]$. For SDEs of the form

$$
\dot{\xi}_{i}=G\left(\xi_{i-2}, \xi_{i-1}, \xi_{i}, \xi_{i+1}, \xi_{i+2}\right)+\sum_{k=0}^{N-1} Q_{i, k} \eta_{k}
$$

(where $\eta_{k}$ are independent white noises and $\boldsymbol{Q}=\left[Q_{i, k}\right]$ is circulant), this task is pursued via the replacements $\xi_{i} \rightarrow \xi^{\mathrm{mf}}$ and $\xi_{i \pm 1}, \xi_{i \pm 2} \rightarrow f\left(\xi^{\mathrm{mf}}, t\right)$ where $f$ is a (deterministic) field to be determined $[34,35]$. In principle, this $f$ depends on the joint probability density, $p_{5}$, of five terraces. This $p_{5}$ satisfies a BBGKY hierarchy, which in principle involves the joint probability densities, $p_{n}$, of $n$ terraces [35].

Since the $\eta_{k}$ are independent, it can be argued that the term $\sum_{k=0}^{N-1} Q_{i, k} \eta_{k}$ should be replaced by $q \eta$ where $q^{2}=\sum_{k=0}^{N-1} Q_{i, k}^{2}=|\boldsymbol{Q}|^{2}$. A formal justification comes from considering the first equation of the BBGKY hierarchy, which is an evolution equation for the TWD, $P(\xi, t)$, where the coefficient of $\partial_{\xi \xi} P(\xi, t)$ is $\frac{1}{2}|\boldsymbol{Q}|^{2}$. 
Thus, (4.1) becomes

$$
\dot{\xi}^{\mathrm{mf}}=G\left(f\left(\xi^{\mathrm{mf}}, t\right), f\left(\xi^{\mathrm{mf}}, t\right), \xi^{\mathrm{mf}}, f\left(\xi^{\mathrm{mf}}, t\right), f\left(\xi^{\mathrm{mf}}, t\right)\right)+q \eta
$$

By comparison of the Fokker-Planck equation for (4.2) with the first equation of the BBGKY hierarchy for (4.1), one obtains a (self-consistent) formula for $f$ [35]. For a linear $G$, such a self-consistent $f$ requires knowing the pair correlation. Specifically, one needs to know the conditional expectation for a terrace width, i.e., the average width of a terrace in a pair of terraces given the value of the width of the other terrace [34].

To circumvent the hurdle of solving the BBGKY hierarchy, it is tempting to apply the decorrelation ansatz $p_{n}\left(\vec{w}_{n}, t\right)=\prod_{j=1}^{n} P\left(w_{j}, t\right)$, where $\vec{w}_{n}=\left(w_{0}, \ldots, w_{n}\right)$ $[34,35]$. For a linear SDE system, i.e., when $G$ is linear, the mean field is given by $f=$ $\mathbb{E} \xi_{i}=0[34,35]$. In $[35]$, where the first-row entries for $\boldsymbol{Q}$ are set to $[2,-1,0 \ldots, 0,-1]$ (yielding a second-order conservative noise scheme similar to (3.5)), it was verified for the linearized SDEs that the long-time limit of the mean field variance coincides with that of the exact solution.

Motivated by these previous studies, we compute the mean field variance for SDEs (2.4) under the $F$-dependent noise (3.14). By taking $f=\mathbb{E} \xi_{i}=0$, these equations are reduced to the effective Langevin equation

$$
\frac{\mathrm{d} \xi^{\mathrm{dc}}}{\mathrm{d} \tau}=-[1-2 p+3 g(1+2 \beta)] \xi^{\mathrm{dc}}+q \widetilde{\eta}(\tau), q^{2}=2 \widetilde{m}_{0}[1-\sqrt{p(1-p)}]
$$

where $\xi^{\mathrm{dc}}$ approximates (in some sense) the mean field stochastic process $\xi^{\mathrm{mf}}$ under the decorrelation ansatz. Thus, $\xi^{\mathrm{dc}}$ is a Gaussian random variable with zero mean 
and variance

$$
\sigma^{\mathrm{dc}}(t)^{2}=\widetilde{m}_{0} \frac{1-\sqrt{p(1-p)}}{1-2 p+3 g(1+2 \beta)}\left\{1-e^{-2 F a[1-2 p+3 g(1+2 \beta)] t}\right\},
$$

which approaches $\sigma^{\mathrm{dc}}(\infty)^{2}=\widetilde{m}_{0}[1-\sqrt{p(1-p)}][1-2 p+3 g(1+2 \beta)]^{-1}$ as $t \rightarrow \infty$.

The long-time limit (4.4) differs from the variance calculated by (3.16). We attribute this discrepancy to the fact that terrace correlations persist at long time (cf. (3.17)), in contradistinction to the decorrelation ansatz. For strong enough step interactions, i.e., $g(1+2 \beta) \gg 1-2 p$, we have $\sigma^{\mathrm{dc}}(\infty)^{2}=\mathcal{O}\left(g^{-1}\right)$ while by (3.17) we have $\mathfrak{C}_{0, \mathrm{st}}=\mathcal{O}\left(g^{-1 / 2}\right)$. Thus, the decorrelation hypothesis exaggerates the narrowing of the TWD; the (positive) terrace width pair correlations favor broadening of the TWD.

\subsection{Related past works and plausible connection to experiments}

Next, we point out features of our predictions for the variances $\mathfrak{C}_{0, \mathrm{st}}$ of $(3.10)$ and (3.16) that may be experimentally testable, and discuss comparisons of these to results of $[10,16]$.

(i) Narrowing of TWD [16]. In the case with a flux $(F)$-dependent noise and large $\nu=a F \varpi^{2} /(2 D)$, by $(3.16)$ the variance $\mathfrak{C}_{0, \mathrm{st}}$ approaches the limit

$$
\mathfrak{C}_{0, \mathrm{st}} \rightarrow \widetilde{m}_{0}(1+4 g)^{-1 / 2} \quad \text { as } \nu \rightarrow \infty
$$

under the assumption that the quasi-steady approximation is meaningful. In the near-equilibrium case with large $\nu$, the variance approaches a constant with complicated $g$-dependence; cf. (3.12). These predictions should be contrasted with the 
corresponding variance in [16], where $\sigma^{2}=\mathfrak{C}_{0, \mathrm{st}}=\mathcal{O}\left(F^{-1}\right)$ for large $F$.

For small deposition flux $F(p \uparrow 1 / 2)$, (3.16) yields $\mathfrak{C}_{0, \mathrm{st}} \rightarrow \mathcal{O}\left(g^{-1 / 2}\right)$ as $\nu \downarrow$ 0 . In the near-equilibrium case, the variance (3.10) is $\mathcal{O}\left(g^{-1}\right)$ as $F \downarrow 0$. These predictions stand in contrast to the behavior $\mathfrak{C}_{0, \mathrm{st}}=\mathcal{O}\left(F^{-2}\right)$ given in [16]. Hence, our noise models significantly tone down the narrowing of the TWD reported in [16], where deposition is the only source of TWD narrowing and the noise is $F$ independent. More precisely, the discrepancy of our result for $\mathfrak{C}_{0 \text {,st }}$ with the variance in [16] is due to: (i) the different scaling of the noise term with $F$; and (ii) the application of the mean field approximation with a non-conservative white noise in [16]. Other models of noise for terraces can plausibly be constructed by mixing elements of (3.5) and (3.14).

(ii) Comparison to [10]. In the model of [10], the deterministic equations account for the same kinetic (flux-induced) asymmetry in step motion but steps are non-interacting. The noise used in [10] for every terrace, $w_{i}$, appears to have the form $\eta_{i, 1} \sqrt{F p w_{i-1}}+\eta_{i, 2} \sqrt{F p w_{i}}+\eta_{i, 3} \sqrt{F(1-p) w_{i}}+\eta_{i, 4} \sqrt{F(1-p) w_{i+1}}$ where $\eta_{i, k}(k=1, \ldots, 4)$ are independent white noises and units with $a=1$ are apparently used. In the mean field approximation (under the decorrelation ansatz for terrace widths), this model yields the (scaled by $\varpi^{2}$ ) steady-state variance $\hat{\sigma}(t \rightarrow \infty)^{2}=(1-2 p)^{-1}[10]$. In [10] this prediction is found to appreciably overestimate the variance produced by kinetic Monte Carlo simulations for small rate $F$ (see figure 4 in [10]). Since the simulations do not allow for step crossing, it is expected that these simulations do include entropic repulsions between steps. 
On the other hand, our analytical model does contain the effect of step repulsion explicitly (via $\breve{g}$ ) and predicts a lower value of the variance for small $F$.

(iii) Experiment on Si(111) [14,43].

Scanning tunneling microscopy (STM) measurements on Si(111) can yield images of "quasi-1D" step trains [43]. These systems have been modeled previously by deterministic 1D evolution equations resembling (2.1) [8]; our stochastic model (2.4) accounts for the additional effects of noise. By measuring terrace width correlations under equilibrium and non-equilibrium conditions, it should be possible to test the validity of the noise models (3.5) and (3.14), respectively.

As mentioned in [16], experimental techniques that enable observation of equilibrium TWD can in principle also probe narrowing due to the combined influence of growth and step interactions. An example is the reflection electron microscopy applied in [14]. In this experiment [14], TWD narrowing is observed on vicinal Si(111) at $1100{ }^{\circ} \mathrm{C}$ and attributed solely to electromigration (which also causes a drift in the adatom flux) although a deposition flux from above and step interactions are present.

\subsection{On the validity of the model}

We repeat that a limitation of our model is due to its $1 \mathrm{D}$ character. Despite this feature, our analysis may be useful in describing quantitative aspects of "quasi1D" step systems, e.g., those in [8,43], as also noted in [35].

Another limitation is related to the perturbation expansion for "small" noise 
(small terrace width fluctuations), which underlies our linearization. Within this approximation, an indication that the linearization may not be valid arises if the TWD variance satisfies $\mathfrak{C}_{0}(t)>1$ : by $(2.10)$, the negative tail of the (approximate) TWD, $P(\xi, t ; N)$, may have an appreciable effect on moments. To provide a condition necessary for the validity of our model, we require that $\mathfrak{C}_{0}(t)$ is sufficiently small for all $t$.

We also comment on the magnitude of the ratio $\nu=F a \varpi^{2} /(2 D)$ which first appears in (2.1). This constant is considered to be $\mathcal{O}(1)$, so that the convection (resulting from our transformation to the step comoving frame) is not necessarily negligible in our analysis. For many physical systems, $\nu$ can be estimated by [8]

$$
\nu=\frac{F a \tau_{0}}{e^{-E_{\mathcal{D}} / T}} \frac{1}{2 \widetilde{m}_{0}^{2}}
$$

where $E_{\mathcal{D}}$ is the energy barrier (i.e. a diffusion barrier) that an adatom must overcome to move to an adjacent lattice site, and $\tau_{0}^{-1}$ is an attempt frequency; we use the relation $D=a^{2} \tau_{0}^{-1} \exp \left[-E_{\mathcal{T}} / T\right]$ [8]. Typical values for $E_{\mathcal{T}}$ range from $0.04 \mathrm{eV}$ for $\mathrm{Al}(111)$ to $0.97 \mathrm{eV} \pm 0.07 \mathrm{eV}$ for $\mathrm{Si}(111)$ [8]. The attempt frequency, $\tau_{0}^{-1}$, is usually taken to be $10^{13} \mathrm{~s}^{-1}$. For a deposition rate of one monolayer per second $\left(F a=1 \mathrm{~s}^{-1}\right)$ at room temperature, values of $\nu$ are found to range approximately from $10^{-11}$ to $10^{8}$. Although we find the upper bound of these values to be exaggerated, we are in principle motivated to account for all $\nu \geq 0$ in our model. For many experimental systems of current interest, however, our model is expected to be in the small-deposition-flux regime, with small $\nu$ and $p \approx 1 / 2$. Notably, an advantage of the model for arbitrary $\nu$ is that it is generic to a variety of asymmetric kinetic 
processes (as noted in chapter 1). We repeat that care must be exercised in applying the long-time limit of (3.15) (see Remark 4).

\subsection{Conclusion}

In this thesis, we studied small stochastic fluctuations of line defects on a crystal surface when material is deposited from above in $1+1$ dimensions. This work has been inspired by $[9,10,16]$, and aims to complement recent kinetic studies in the steady-state distribution of terrace widths on vicinal crystal surfaces [34-36]. We started with a reasonably general formulation of terrace width fluctuations in 1+1 dimensions, allowing for a noise that stems from the fluctuation-dissipation theorem for adatoms [9]; and, alternatively, from a phenomenological description of a deposition-dependent effect at step edges [10]. The ensuing models include a conservative noise, deposition-flux-induced kinetic asymmetry in the noise coefficients, and step repulsion.

Our perturbation analysis led to a Gaussian TWD and a simple closed form for the associated variance. Similarly, we obtained closed-form expressions for the terrace width covariance matrices. The TWD, which is symmetric about the expectation of the terrace width, is plausibly valid for values of terrace widths near the peak of the actual TWD. On the basis of these results, we inferred that growth combined with step interaction sustain a reduced narrowing of the TWD with the deposition rate, $F$, in juxtaposition to the corresponding (more exaggerated) F-dependence noted in [16]. Furthermore, we applied a previous mean field approach [16,34], and 
thereby indicated and discussed the role of terrace width pair correlations at long times. We found that these correlations decay exponentially in the height difference between two terraces, and practically vanish in the macroscopic limit.

Our analysis points to several open questions. For example, the nonlinearities left out from our stochastic scheme should cause the TWD to be non-symmetric about the mean [35]. The derivation of such a modified TWD remains unresolved. Any effect of multiplicative noise (where the noise coefficients depend on terrace widths) is left for future work. Another open question concerns 2D geometries, which were not considered here. In real systems, edge atoms diffuse along steps (besides diffusing on terraces and attaching/detaching at step edges). In addition, kinks on steps influence the form of noise. Many-step interacting systems in 2D are the subject of work in progress. 


\section{Appendix A}

Derivations for covariant matrix

In this appendix, we derive (2.8) and use this result to show (2.11). In the following, we abandon the summation convention for repeated indices.

Lemma A.1. For arbitrary $N \times N$ circulant matrices $\boldsymbol{A}$ and $\boldsymbol{Q}$, and a stochastic process $\boldsymbol{\xi}(t)$ given by (2.7) with $\boldsymbol{\xi}(0)=0$, the covariance matrix $\mathfrak{C}_{m}(t)$ is given by

Proof. Consider a given lattice site with index $j$. By the invariance of (2.4) under translations of $j$, the covariance matrix is

$$
\begin{array}{r}
\mathfrak{C}_{m}(t)=N^{-1} \sum_{j=0}^{N-1} \mathbb{E}\left[\xi_{j}(t) \xi_{j+m}(t)\right]=N^{-1} \mathbb{E}\left[\boldsymbol{\xi}(t)^{T}(\boldsymbol{\xi}(t))_{m}^{c}\right] \\
=\frac{1}{N} \int_{0}^{\tau} \mathrm{d} s \int_{0}^{\tau} \mathrm{d} s^{\prime} \mathbb{E}\left[\left(\boldsymbol{Q} e^{-\boldsymbol{A}(\tau-s)} \boldsymbol{\eta}(s)\right)^{T}\left(\boldsymbol{Q} e^{-\boldsymbol{A}\left(\tau-s^{\prime}\right)} \boldsymbol{\eta}\left(s^{\prime}\right)\right)_{m}^{c}\right],
\end{array}
$$

where the symbol $(\cdot)_{m}^{c}$ denotes the $m$ th cyclic permutation of the vector inside the parenthesis. For any circulant matrix $\boldsymbol{X}$ and vector $\boldsymbol{y}$, the $i$ th component of $(\boldsymbol{X} \boldsymbol{y})_{m}^{c}$ is

$$
\begin{array}{r}
{\left[(\boldsymbol{X} \boldsymbol{y})_{m}^{c}\right]_{i}=\sum_{j=0}^{N-1} X_{i+m, j} y_{j}=\sum_{j=0}^{N-1} X_{i+m, j+m} y_{j+m}} \\
=\sum_{j=0}^{N-1} X_{i, j} y_{j+m}=\left[\boldsymbol{X}(\boldsymbol{y})_{m}^{c}\right]_{i}
\end{array}
$$

where all indices are interpreted as modulo $N$, and the last line follows from the definition of a circulant matrix. Moreover, the product of any two circulant matrices 
is circulant. Hence, the term $\boldsymbol{Q} \exp \left[-\boldsymbol{A}\left(\tau-s^{\prime}\right)\right]$ appearing in (A.1) is circulant. Equation (2.8) is deduced from (A.2).

Next, we derive (2.11) on the basis of (2.8). This amounts to proving the following.

Proposition A.1. For arbitrary $N \times N$, constant circulant matrices $\boldsymbol{A}$ and $\boldsymbol{Q}$, and delta-correlated noise $\eta(s)$, where $\mathbb{E}\left[\eta(s) \eta\left(s^{\prime}\right)\right]=\delta\left(s-s^{\prime}\right)$, the following relation holds:

$$
\begin{gathered}
\mathbb{E}\left[\boldsymbol{\eta}\left(s^{\prime}\right)^{T} e^{-\boldsymbol{A}^{T}\left(\tau-s^{\prime}\right)} \boldsymbol{Q}^{T} \boldsymbol{Q} e^{-\boldsymbol{A}(\tau-s)}\left(\boldsymbol{\eta}(s)_{m}^{c}\right)\right]= \\
\delta\left(s-s^{\prime}\right) \sum_{k=0}^{N-1} \vartheta_{k} e^{-\lambda_{k}(\tau-s)} \cos \left[\frac{2 \pi k m}{N}\right]
\end{gathered}
$$

where $\theta_{k}$ are the eigenvalues of $\boldsymbol{Q}^{T} \boldsymbol{Q}$ and $\lambda_{k}$ are the eigenvalues of $\boldsymbol{A}+\boldsymbol{A}^{T}$.

Proof. Since $\boldsymbol{A}$ and $\boldsymbol{Q}$ are circulant, they are diagonalized by the discrete Fourier transform $\boldsymbol{F}$, where $F_{k, l}=e^{-2 \pi i(k l / N)}\left(i^{2}=-1\right)$. Specifically,

$$
e^{-\boldsymbol{A}^{T}\left(\tau-s^{\prime}\right)} \boldsymbol{Q}^{T} \boldsymbol{Q} e^{-\boldsymbol{A}(\tau-s)}=\boldsymbol{F}^{-1} \boldsymbol{D} \boldsymbol{F}, \quad \mathcal{D}_{j, k}=\delta_{j, k} \vartheta_{k} e^{-\lambda_{k}(\tau-s)}
$$

which follows from the relation $\boldsymbol{F}^{-1} \exp (\boldsymbol{C}) \boldsymbol{F}=\exp \left(\boldsymbol{F}^{-1} \boldsymbol{C F}\right)$ for any matrix $\boldsymbol{C}$. Hence, the expectation of the left-hand side of (A.3) simplifies to

$$
\begin{gathered}
\mathbb{E}\left[\boldsymbol{\eta}\left(s^{\prime}\right)^{T} \boldsymbol{F}^{-1} \mathcal{D} \boldsymbol{F}(\boldsymbol{\eta}(s))_{m}^{c}\right]=\frac{1}{N} \sum_{j, k, n=0}^{N-1} \mathbb{E}\left[\eta_{j}\left(s^{\prime}\right) e^{2 \pi i j k / N} \mathcal{D}_{k, k} e^{-2 \pi i k n / N}\left[(\eta(s))_{m}^{c}\right]_{n}\right] \\
=\frac{\delta\left(s-s^{\prime}\right)}{N} \sum_{j, k, n=0}^{N-1} \mathcal{D}_{k, k} e^{2 \pi i k(j-n) / N} \delta_{j+m, n}=\delta\left(s-s^{\prime}\right) \sum_{k=0}^{N-1} \mathcal{D}_{k, k} e^{-2 \pi i k m / N}
\end{gathered}
$$

where we have written sums explicitly to avoid any ambiguity when summing over $k$. 
Furthermore, note that the eigenvalues of a circulant matrix $\boldsymbol{C}$ are given by the product $F_{j, k} C_{k, 0}$. Since $\boldsymbol{A}+\boldsymbol{A}^{T}$ and $\boldsymbol{Q} \boldsymbol{Q}^{T}$ are both symmetric and circulant, they satisfy $C_{k, 0}=C_{N-k, 0}$. This last property implies (A.3).

Proposition A.1 offers an extension of a result given in [35]. More precisely, in the case of the TWD variance, i.e., with $m=0$, we recover formula (16) given in $[35]$.

\section{A.1 Useful relations}

This appendix provides some relations that help simplify results of section 2 .

\section{A.2 Long-time behavior of integral (2.13)}

We show that the exponential term in (2.13) vanishes when $\tau \rightarrow \infty$. This property holds for both forms of $\vartheta(x)$ given in section 3 , and the $\lambda_{k}$ given by (3.7) when $F \neq 0$. Specifically, the following inequality holds:

$$
\begin{array}{r}
\left|\int_{0}^{1} \mathrm{~d} y \vartheta(y) \lambda^{-1}(y) e^{-\tau \lambda(y)} \cos (2 \pi m y)\right| \leq c_{1} \int_{0}^{1} \mathrm{~d} y e^{-\tau c_{2}(1-\cos (2 \pi y))} \\
<c_{1} \int_{-\infty}^{\infty} e^{-\tau c_{2} y^{2}} \mathrm{~d} y \\
=\mathcal{O}\left(\tau^{-1 / 2}\right) \quad \text { as } \tau \rightarrow \infty,
\end{array}
$$

where $c_{1}, c_{2}>0$ are constants independent of $y$. 


\section{A.3 Two formulas}

In certain cases, the time dependence of the covariance matrix, e.g., in (3.18), can be resolved via the identity [42]

$$
e^{z \cos (x)}=I_{0}(z)+2 \sum_{n=1}^{\infty} I_{n}(z) \cos (n x)
$$

where $I_{n}(z)$ is the modified Bessel function of order $n$.

The following integral, when combined with the appropriate trigonometric identities, is useful for calculating steady-state covariances by (2.8):

$$
\int_{0}^{\pi} \mathrm{d} x \frac{\cos (n x)}{1-\alpha \cos (x)}=\frac{\pi}{\sqrt{1-\alpha^{2}}}\left[\frac{1-\sqrt{1-\alpha^{2}}}{\alpha}\right]^{|n|}
$$

where $|n|=0,1,2, \ldots$. This formula can be shown by contour integration or, alternatively, it can be found in integral tables, e.g., [44].

\section{A.4 Time-dependent covariance for $\nu \ll 1$}

In this appendix, we asymptotically evaluate integral (3.15) for small $\nu=$ $F a \varpi^{2} /(2 D), g=\mathcal{O}(1)$, and $m=\mathcal{O}(1)$. For algebraic convenience, set $\widetilde{m}_{0}=1$ (only in this appendix).

By the change of the integration variable to $z=\sin (\phi / 2)$, the integral becomes

$$
\begin{aligned}
\mathfrak{C}_{m}(t)=\frac{1}{2 \pi} \frac{1}{g(1+2 \beta)} & \int_{0}^{1} \frac{\mathrm{d} z T_{m}\left(1-2 z^{2}\right)}{\sqrt{1-z^{2}}}\left[1+2 \sqrt{p(1-p)}\left(1-2 z^{2}\right)\right] \\
& \times \frac{1-e^{-\left(\alpha_{1}^{2}+z^{2}\right) \alpha_{2}^{2} z^{2}}}{z^{2}+\alpha_{1}^{2}}
\end{aligned}
$$

where $T_{m}(x)$ is the $m$ th-order Chebyshev polynomial of the first kind, $\alpha_{1}^{2}=(1-$ $2 p)[4 g(1+2 \beta)]^{-1}$, and $\alpha_{2}^{2}=16 \operatorname{tg} F a(1+2 \beta)$. As $\nu \downarrow 0$ with fixed $g$ and $t F a \geq \mathcal{O}(1)$, 
we have $\alpha_{1}^{2}=\mathcal{O}\left(\nu^{2}\right)$ while $\alpha_{2}^{2} \geq \mathcal{O}\left(\nu^{-1}\right)$. Thus, the task is to evaluate $\mathfrak{C}_{m}(t)$ by (A.9) for small $\alpha_{1}$ and large $\alpha_{2}$ (where $\left.\alpha_{1}, \alpha_{2}>0\right)$.

By inspection of the integrand in (A.9), we distinguish the following cases.

(i) $\alpha_{2}^{-1} \gg \alpha_{1}^{2}$, i.e., $t F a \ll g(1+2 \beta)(1-2 p)^{-2}$ : The major contribution to integration comes from a neighborhood of width $\mathcal{O}\left(\alpha_{2}^{-1 / 2}\right)$ around $z=0$, and $\alpha_{1}^{2}$ is neglected compared to $z^{2}$. Specifically, we have

$$
\begin{aligned}
\mathfrak{C}_{m}(t) & \approx \frac{1}{\pi} \frac{1}{g(1+2 \beta)} \int_{0}^{1} \sqrt{1-z^{2}} \frac{1-e^{-\alpha_{2}^{2} z^{4}}}{z^{2}} \mathrm{~d} z \\
& =\frac{1}{\pi} \frac{1}{g(1+2 \beta)}\left(\int_{0}^{A}+\int_{A}^{\alpha_{2}^{2}}\right) \int_{0}^{1} \sqrt{1-z^{2}} z^{2} e^{-y z^{4}} \mathrm{~d} z \mathrm{~d} y
\end{aligned}
$$

where $A$ is any fixed yet large positive number and $T_{m}\left(1-2 z^{2}\right) \approx 1$ to leading order. Thus, the $y$ in $\int_{A}^{\alpha_{2}^{2}}$ is large and the respective integral in $z$ is evaluated by expanding the integrand near $z=0$. Finally, we obtain

$$
\mathfrak{C}_{m}(t) \approx \frac{2}{\pi} \Gamma\left(\frac{3}{4}\right)[g(1+2 \beta)]^{-3 / 4}(t F a)^{1 / 4}
$$

where $\Gamma(\zeta)$ is the Gamma function [45]. So, if $t F a$ is fixed as $\nu \downarrow 0$, then $\mathfrak{C}_{m}(t)$ vanishes as $\mathcal{O}\left(\nu^{3 / 4}\right)$. However, if instead $2 D t / \varpi^{2}$ is kept fixed as $\nu \downarrow 0$, then $\mathfrak{C}_{m}(t)=$ $\mathcal{O}(\nu)=\mathcal{O}(F)$

(ii) $\alpha_{2}^{-1} \ll \alpha_{1}^{2}$, i.e., $t F a \gg g(1+2 \beta)(1-2 p)^{-2}$ : The major contribution to integration in the time-dependent term of (A.9) arises from a vicinity of width $\mathcal{O}\left(\left(\alpha_{1} \alpha_{2}\right)^{-1}\right)=$ $\mathcal{O}\left((\nu t F a)^{-1 / 2}\right)$ around $z=0$. Approximating $T_{m}\left(1-2 z^{2}\right) \approx 1$, we obtain

$$
\mathfrak{C}_{m}(t) \approx \frac{1}{2 \pi} \frac{1}{g(1+2 \beta)} \int_{-1}^{1} \frac{\sqrt{1-z^{2}}}{z^{2}+\alpha_{1}^{2}} \mathrm{~d} z
$$

To evaluate this integral to leading order in $\nu$, we apply analytic continuation of the integrand to complex $z$. So, deform the path of integration to the upper half of 
the complex $z$-plane so as to pick up the residue from the simple pole at $z=i \alpha_{1}$ $\left(i^{2}=-1\right)$. Thus, compute

$$
\mathfrak{C}_{m}(t)=(2 g / 3)^{-1 / 2}+\mathcal{O}(\nu)=\mathfrak{C}_{m}(t \rightarrow \infty ; p \uparrow 1 / 2, g)
$$

which is the limit of steady-state formulas (3.16) and (3.17) for small $\nu=F a \varpi^{2} /(2 D)$. 


\section{Bibliography}

[1] Spohn H 1991 Large Scale Dynamics of Interacting Particles (Berlin: Springer)

[2] Dolbow J, Khaleel M A, and Mitchell J 2004 Multiscale Mathematics Initiative: A Roadmap Technical Report PNNL 14966 (Richland, WA: Pacific Northwest National Laboratory, U.S. Department of Energy)

[3] Boltzmann L 1864 Lectures on Gas Theory (New York: Dover)

[4] Kac M 1956 Proceedings of the Third Berkeley Symposium on Mathematical Statistics and Probability 3 ed J Neuman (Berkeley, CA: University of California Press) p 171

[5] Cercignani C, Illner R, and Pulvirenti M 1994 The Mathematical Theory of Dilute Gases (New York: Springer)

[6] Pulvirenti M 1996 in Probabilistic Models for Nonlinear Partial Differential Equations eds D Talay and L Tubaro (Berlin: Springer) p 96

[7] Michely T and Krug J 2004 Islands, Mounds and Atoms: Patterns and Processes in Crystal Growth Far From Equilibrium (Berlin: Springer)

[8] Jeong H C and Williams E D 1999 Surf. Sci. Rep. 34171

[9] Pierre-Louis O and Misbah C 1998 Phys. Rev. B 582259

[10] Williams D R M and Krishnamurthy M 1993 Appl. Phys. Lett. 621350

[11] Burton W K, Cabrera N and Frank F C 1951 Philos. Trans. R. Soc. London Ser. A 243299

[12] Marchenko V I and Parshin A Ya 1980 Sov. Phys. JETP 52129

[13] Najafabadi R and Srolovitz D J 1994 Surf. Sci. 317221

[14] Cohen S D, Schroll R D, Einstein T L, Métois J-J, Gebremariam H, Richards H L, and Williams E D 2002 Phys. Rev. B 66115310

[15] Einstein T L 2003 Ann. Henri Poincaré 4 S811 
[16] Ben-Hamouda A, Pimpinelli A, and Einstein T L 2009 Europhys. Lett. 8826005

[17] Uwaha M and Saito Y 1992 Phys. Rev. Lett. 68224

[18] Ihle T, Misbah C, and Pierre-Louis O 1998 Phys. Rev. B 582289

[19] Gossmann H-J, Sinden F W, and Feldman L C 1990 J. Appl. Phys. 67745

[20] Tokura Y, Saito H, and Fukui T 1989 J. Cryst. Growth 9446

[21] Dufay M, Frisch T, and Debierre J-M 2007 Phys. Rev. B 75 241304(R)

[22] Ghez R and Iyer S S 1988 IBM J. Res. Develop. 32804

[23] Ehrlich G and Hudda F 1966 J. Chem. Phys. 441039

Schwoebel R L and Shipsey E J 1966 J. Appl. Phys. 373682

[24] Krug J 1997 in Dynamics of Fluctuating Interfaces and Related Phenomena eds D Kim, H Park and B Kahng (Singapore: World Scientific) p 95

[25] Biehl M, Ahr M, Kinne M, Kinzel W, and Schinzer S 2001 Phys. Rev. B 64 113405

[26] Slanina F, Krug J, and Kotrla M 2005 Phys. Rev. E 71041605

[27] Krug J and Schimschak M 1995 J. Phys. I (France) 51065

[28] Stoyanov S S, Ichikawa M, and Doi T 1993 Jpn. J. Appl. Phys. 322047

[29] Stoyanov S S 1991 Jpn. J. Appl. Phys. 301

[30] Liu D-J and Weeks J D 1998 Phys. Rev. B 5714891

[31] Krug J 2005 in Multiscale Modeling of Epitaxial Growth ed A Voigt (Basel: Birkhäuser) p 69

[32] Krug J 2008 Europhys. Lett. 60788

[33] Ben-Hamouda A, Absi N, Hoggan P E, and Pimpinelli A 2008 Phys. Rev. B 77245430

[34] Margetis D 2010 J. Phys. A: Math. Theor. 43065003 
[35] Patrone P N, Einstein T L, and Margetis D 2010 Phys. Rev. E 82061601

[36] Margetis D, Patrone P N, and Einstein T L 2011 Materials Research Society (MRS) Proceedings 1318, mrsf10-1318uu07-04; doi:10.1557/opl.2011.269 (Cambridge University Press); http://journals.cambridge.org/action/displayAbstract?fromPage=online\&aid $=8200644 \& f$ Type=RA\&fileId=S1946427411002697

[37] Evans J W, Thiel P A, and Bartelt M C 2006 Surf. Sci. Rep. 611

[38] Pimpinelli A and Villain J 1998 Physics of Crystal Growth (Cambridge, UK: Cambridge University Press)

[39] Misbah C, Pierre-Louis O, and Saito Y 2010 Rev. Mod. Phys. 82981

[40] Atzberger P 2010 J. Comp. Phys. 2293474

[41] Grenander U and Szegö G 1958 Toeplitz Forms and Their Applications (Berkeley, CA: University of California Press)

[42] Bateman Manuscript Project 1953 Higher Transcendental Functions vol II ed A Erdélyi (New York: McGraw-Hill)

[43] Fu E S, Johnson M D, Liu D-J, Weeks J D, and Williams E D 1996 Phys. Rev. Lett. 771091

[44] Gradshteyn I S and Ryzhik I M 1994 Tables of Integrals, Series, and Products ed A Jeffrey (New York: Academic Press, 5th edition)

[45] Bateman Manuscript Project 1953 Higher Transcendental Functions vol I ed A Erdélyi (New York: McGraw-Hill) 